\title{
Influence of temperature and cooling liquid immersion on the mechanical behavior of a PPS composite: experimental study and constitutive equations
}

\author{
Quentin C. P. Bourgogne ${ }^{1,2}$ (D) Vanessa Bouchart $^{1} \cdot$ Pierre Chevrier $^{1} \cdot$ Emmanuel Dattoli $^{2}$
}

Received: 16 September 2019 / Accepted: 3 February 2020 / Published online: 10 February 2020

(c) Springer Nature Switzerland AG 2020

\begin{abstract}
This paper presents an experimental and theoretical study conducted on a PolyPhenylene Sulfide with 40 wt.\% of short glass fibers (PPS GF40) and its matrix. Widely used for under-the-hood applications in the automotive industry, this material is subjected to high temperatures and to the ageing effects of the cooling liquids. Therefore, the consideration of those conditions is essential to design mechanical parts. Thus, an experimental campaign in the tensile mode was carried out at different temperatures and for various glycol proportions in the cooling liquid, under monotonic and cyclic loadings on neat and reinforced PPS. The results of these tests enabled us to highlight some of the main physical phenomena occurring during these experiments under tough hydrothermal conditions. Following this analysis, a visco-elasto-pseudo-viscoplastic model is proposed. Moreover, this model enables the consideration of the effects of the cooling liquid and its constituents using a temperature/humidity equivalence. This model also takes into account the consequences of the glass transition on the mechanical behavior of the material. Its accuracy was confronted to that of an artificial-intelligence-based model, so as to know what the physically possible maximum accuracy is. Finally, the evolution of the model parameters was studied with the adjunction of short glass fibers and for various orientation distributions of those fibers.
\end{abstract}

Keywords Polymer matrix composites · Thermomechanical · Ageing · Analytical modelling · Mechanical testing · Sequential model

\section{Introduction}

Environmental issues are one of the main problematics to take into account for new industrial developments and especially for car manufacturers aiming at reducing costs and $\mathrm{CO}_{2}$ emissions. One way to respond to these challenges is to reduce the weight of the parts [1]. In order to take into account industrial, economic and environmental issues, the use of short-glass-fiber-reinforced thermoplastics is considerably increasing, including in the design of under-the-hood components. These parts are subjected to elevated temperatures that can reach $130^{\circ} \mathrm{C}$ and are fully immersed in the cooling liquid when activated. Those conditions have a strong impact on the mechanical properties of plastic composites such as polyamides [1-4]. Even though polyamide is one of the most common matrix used in the automotive industry, this material may not be strong enough for some dynamic parts supporting important loads within this environment. That is why the study presented in this paper was conducted using a PPS, which has more resistance to chemical products and to temperature $[5,6]$.

Quentin C.P. Bourgogne, quentin.bourgogne@univ-lorraine.fr| ${ }^{1}$ Laboratoire d'Etude des Microstructures et de Mécanique des Matériaux (LEM3), Université de Lorraine, CNRS, Arts et Métiers ParisTech, 57000 Metz, France. ${ }^{2}$ Pierburg Pump Technology, 1 rue Denis Papin, ZA, Kickelsberg, 57970 Basse-Ham, France. 
PolyPhenylene Sulfide (PPS) is a semi-crystalline polymer classified as a high-performance thermoplastic and is thus used for applications in a wide range of fields, from electronics to chemical sectors and aerospace, due to its tribological properties and its easy injection [7-15]. PPS parts are commonly blended with short fibers to improve their mechanical behavior $[16,17]$. In order to design the parts in hydrothermal conditions and to build a predictive model, the complexity of the material and of the environment has to be fully taken into account. The adjunction of fibers induces more complexity in the understanding of the material's properties since the fiber ratio and the orientation of these fibers influence the mechanical behavior of the composite [18]. As some of the main phenomena are occurring on the matrix, its behavior must be closely monitored. Indeed, the temperature and the ageing process have amplified effects on unreinforced materials compared to reinforced materials [19], thanks to a structural modification appearing in the polymer. This is due to an intensification of the molecular chain motion as the temperature increases. This modification is even higher above the glass transition temperature $\mathrm{Tg}$, which is a reference temperature that constitutes a limit for many constitutive models. The mechanical behavior of polymers is so different under and above $\mathrm{Tg}$ that some authors developed constitutive models for neat polymers, that are valid either below or above this temperature $[20,21]$. The influence of the relative humidity on the mechanical response is known to be very strong on polyamides and their derivatives [22, 23] due to the "plasticizing" effect of water [24]. Indeed, water molecules are absorbed by amorphous phases [3], which induces a variation of the glass transition temperature [25]. Then, considering our fully immersed environment and the large range of temperatures to factor in, the cited phenomenological models $[2,20$, $21,23,26-28]$ are presenting some limitations for our applications. Indeed, those models cannot deal with the extrapolation of mechanical properties of aged materials immersed in a two-constituent liquid. Hence, the comparison by extrapolation is not possible. Some models can deal with the influence of the temperature but they are developed in the framework of micromechanics. The micromechanics-based models are dealing with microparameters like the molecular chain motion. This kind of parameters calls for specific tests and cannot easily be performed in an industrial context. It is therefore beyond the scope of the study.

This paper aims at defining the characterization and a phenomenological model for a neat PPS, taking into account the thermal and ageing effects using a rheological scheme with the minimum parameters, in order to define an easier way of dimensioning PPS parts in industry. Moreover, as this study takes place in an industrial context, the experimental data are extracted as much as possible from tensile tests, which can be reproduced with industrial equipment quite easily. Monotonic and cyclic tensile tests were therefore performed on aged samples and at different temperatures in order to study the evolution of the coupled viscosity-damaging impact through cycles and temperatures and to prove that the parameters of the models are linked to physical phenomena. Finally, the model was applied to composite experimental data and a quick study of the evolution of the model parameters as a function of the fiber ratio and the fiber orientation was performed.

\section{Experiments}

The experimental protocol starts with the extraction of the samples and, for some of them, an ageing process was set up before performing the tensile tests, in order to study the effect of liquid absorption on the mechanical properties.

\subsection{Materials and specimen}

Two materials were used in this study. One is a semi-crystalline polyphenylene sulphide with a $40 \%$ massive fraction of glass fiber reinforcement (PPSGF40). The other one is its matrix (PPS). Both were provided by Solvay and are respectively commercialized under the names Ryton ${ }^{\circledR} \mathrm{R}-4$ 200 and Ryton ${ }^{\circledR}$ QA 200 P. Some of their physical properties are listed in Table 1.

$100 \times 100 \times 3.2 \mathrm{~mm}$ square plates were molded by injection at a temperature of $136^{\circ} \mathrm{C}$ and then kindly supplied by Solvay. Dog-bone tensile specimens were made using water jet cutting according to the geometry presented in Fig. 1. The locations of the specimens on the plates are shown in Fig. 2, in order to prevent end effects such as capillarity at the surface of the mold and to obtain
Table 1 Materials used for the experimental study

\begin{tabular}{llllll}
\hline Material & Matrix & Reinforcement & density $(\mathrm{g} / \mathrm{cC})^{*}$ & $\mathrm{Tg}\left({ }^{\circ} \mathrm{C}\right)^{*}$ & $\mathrm{Tm}\left({ }^{\circ} \mathrm{C}\right)^{*}$ \\
\hline Ryton $^{\circledR}$ QA 200 P & Polyphenylene sulfide & None & 1.34 & 90 & 280 \\
Ryton ${ }^{\circledR}$ R-4-200 & Polyphenylene sulfide & 40\% short glass fiber & 1.68 & 90 & 285 \\
\hline
\end{tabular}

*These data were provided by Solvay 

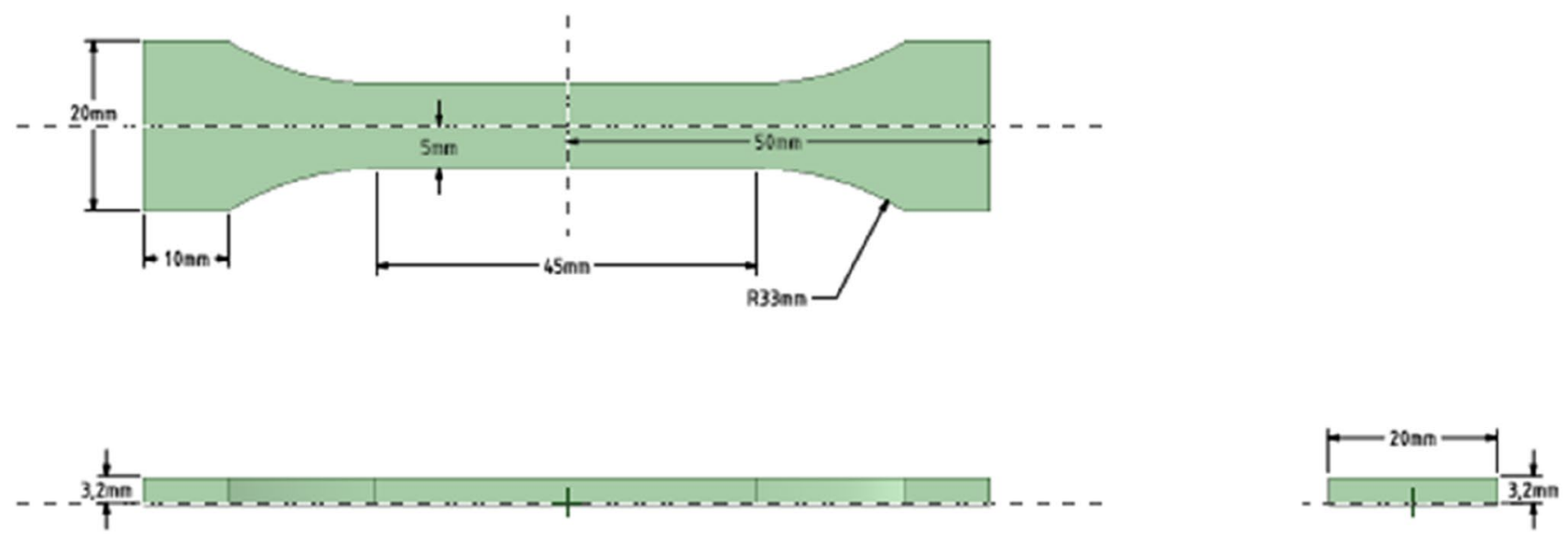

Fig. 1 Specimen geometry

composite samples with different fiber orientations. The matrix being more sensitive to end effects than the composite, the matrix samples were cut transversally to the moldflow direction, allowing the extraction of three samples per plate. The highest stiffness of the composite is reached when the fibers are aligned with the direction of the solicitation. The corresponding samples are then less sensitive to end effects, especially as the working length $L_{0}$ is far from the ends. Consequently, those effects can be neglected. Thus, four samples with longitudinally aligned fibers can be extracted per plate. Shaded areas are unused areas.

\subsection{Ageing method}

PPS is often used in refrigeration systems. In the automotive industry, many different cooling liquids are used. Their composition and proportions, however, are mostly the same. Indeed, those liquids are commonly composed of $50 \%$ of water and $50 \%$ of glycol but for cost-saving reasons and because of the variety of selling areas, car manufacturers can reduce the proportion of glycol. This specific constituent is itself composed of about $90 \%$ of monoethylene glycol, the remaining $10 \%$ representing the additives used by each carmaker. In order to take into account the possibility of different behaviors depending on the glycol proportion, several configurations were tested on the Ryton ${ }^{\circledast}$ QA 200 P matrix. The samples were then immersed in containers for $400 \mathrm{~h}$, in a stove maintained at $90^{\circ} \mathrm{C}$, as shown in Fig. 3. The cooling liquid in the containers was composed from $100 \%$ of water to $100 \%$ of glycol.

The samples were weighed with a precision scale, in a protected environment, with a precision of $0.1 \mathrm{mg}$. These measures have shown that, as glycol has a higher density than water, the more the water proportion is high in the cooling liquid, the more the material gains mass (Fig. 4).
The evolution of absorption can be described by a Fick's law [29] which can be expressed as follows:

$\frac{M_{t}}{M_{m}}=\frac{4}{\sqrt{\pi}}\left[\frac{D t}{h^{2}}\right]^{\frac{1}{2}}$ for $\frac{D t}{h^{2}}<0.05$

$\frac{M_{t}}{M_{m}}=1-\frac{8}{\pi^{2}} \exp \left[-\left[\frac{D t}{h^{2}}\right] \pi^{2}\right]$ for $\frac{D t}{h^{2}}>0.05$

where $t$ is time, $h$ is the thickness of the sample, $D$ is the diffusion coefficient and $\mathrm{Mm}$ is the maximum liquid absorption.

Studying the dynamic of the absorption depending on the liquid's composition shows that the maximum absorption reachable $\mathrm{Mm}$ is the highest when the liquid is fully composed of water (Fig. 5). As the glycol proportion increases, the maximum absorption decreases linearly. Additionally, the diffusion coefficient, which also decreases as the glycol proportion increases, seems to decrease following the Eq. (2):

$D=D_{0} e^{-\frac{a g(\%)}{b}}$

where $D_{0}$ is the maximum diffusion coefficient, obtained in a liquid fully composed of water, $g(\%)$ is the glycol proportion in percentage and $a$ and $b$ are coefficients.

The density of glycol could be responsible for the low behavior. Tensile tests were then performed to determine if the mechanical behavior of aged samples evolved in the same way as the absorption.

\subsection{Tensile tests}

Tensile loads were applied by an INSTRON ${ }^{\mathrm{TM}} 5585 \mathrm{H}$ equipped with mechanical grips, and controlled by a 


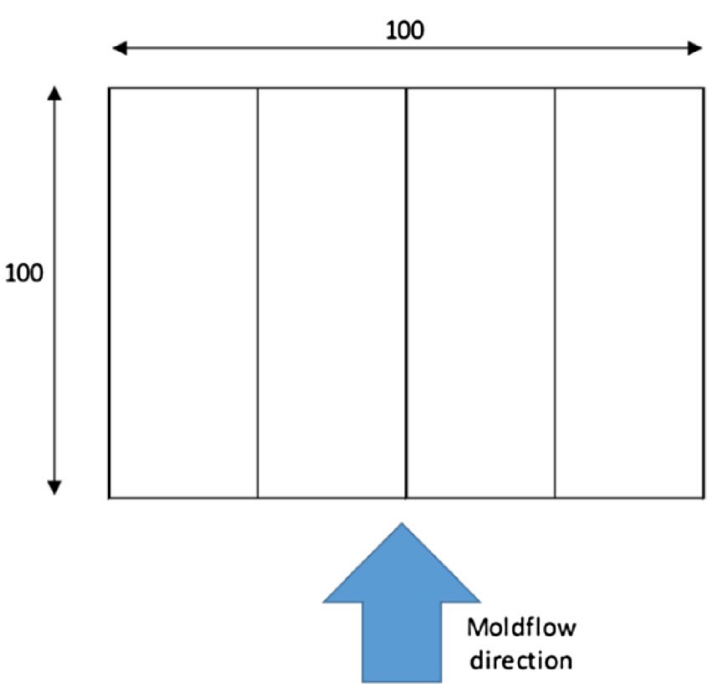

(a)

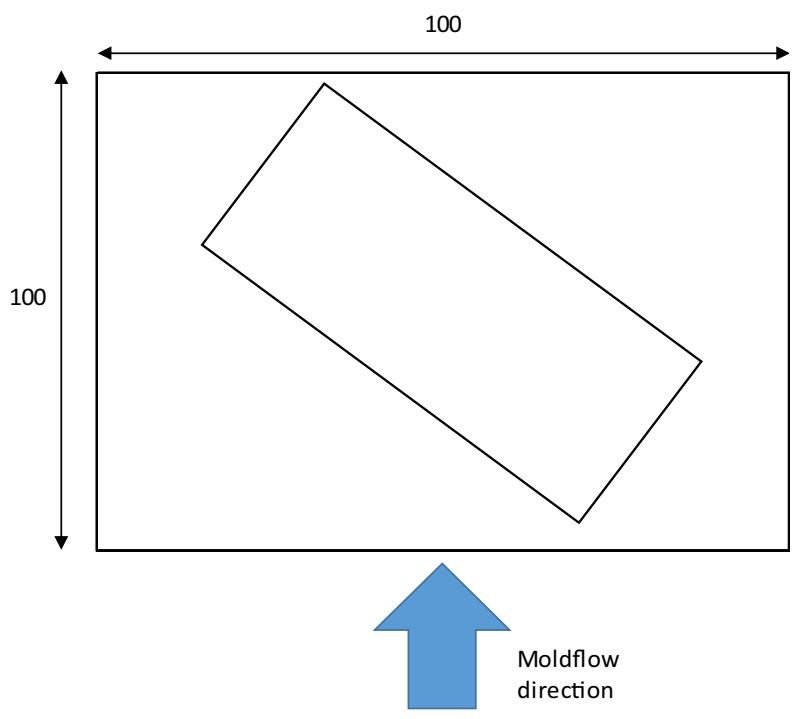

(b)

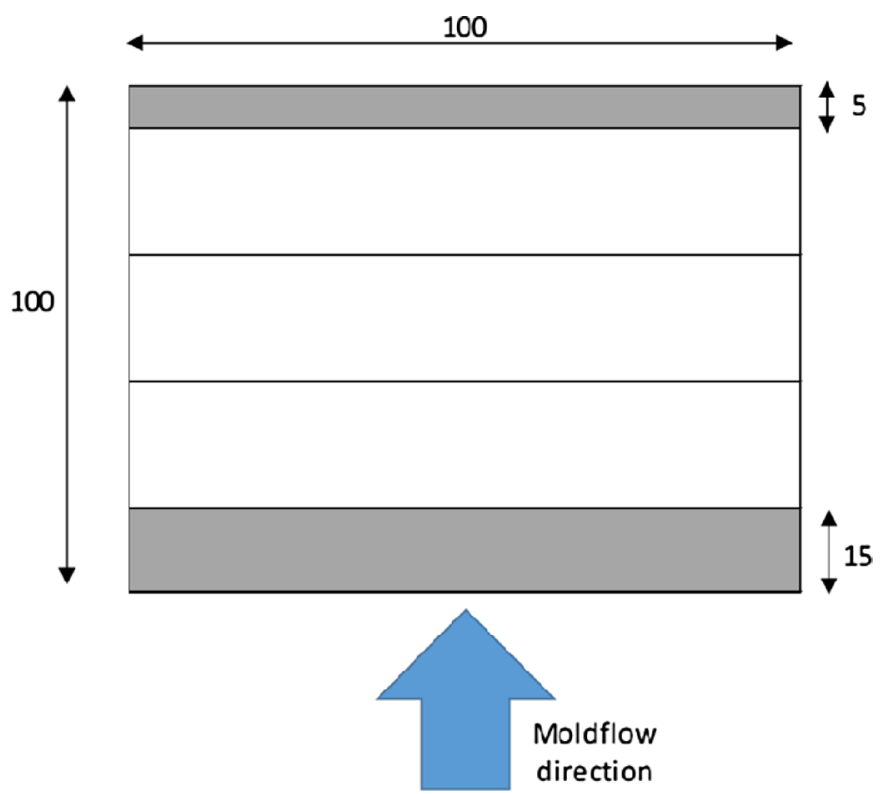

(c)

Fig. 2 a Location of specimen cut from injection plates in the flow direction of PPS GF40. $\mathbf{b}$ Location for $45^{\circ}$ of fiber orientation of PPS GF40. c Location of specimen for PPS and transversally to the moldflow direction for PPS GF40

$10 \mathrm{kN}$-capacity load cell. The local strain was measured with a video extensometer.

Several environmental conditions were tested. Tests at room temperature were performed in standard ISO527-2 conditions. The tests were conducted in a climate chamber, for temperatures varying from 50 to $150{ }^{\circ} \mathrm{C}$. For the tests on aged samples, the specimens were fully immersed for $400 \mathrm{~h}$ so as to ensure the saturation, and then taken out of the container right before submitting them to the tensile test to avoid any loss of cooling liquid in the specimen.

The purpose of the study being the definition of a constitutive model able to predict the behavior of PPS under any condition throughout the service life of the vehicles, a total of eleven different scenarios were tested for the composite and for the matrix: 


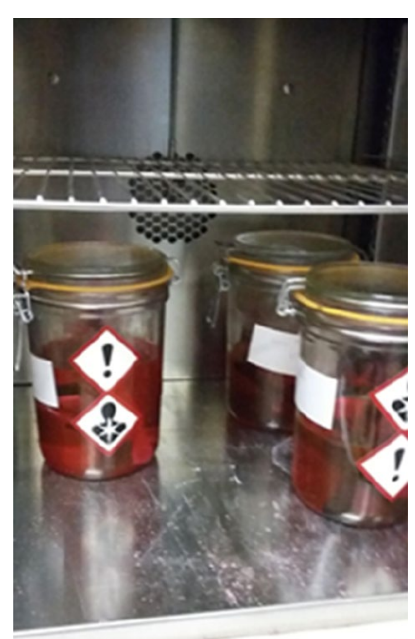

- Five configurations at room temperature $\mathrm{T}=23^{\circ} \mathrm{C}$ for a dry-as-molded (DAM) material and an aged material, fully immersed in multiple coolant liquids (water and glycol) composed of $100 \%$ of water, $25 \%$ of glycol, $50 \%$ of glycol, $75 \%$ of glycol and $100 \%$ of glycol respectively.

- Six configurations at a temperature of $\mathrm{T}=23^{\circ} \mathrm{C}, 50^{\circ} \mathrm{C}$, $70^{\circ} \mathrm{C}, 110^{\circ} \mathrm{C}, 130^{\circ} \mathrm{C}$ and $150^{\circ} \mathrm{C}$ respectively for a DAM material;

All of these configurations were studied using different mechanical tests illustrated in Fig. 6 . Each test was repeated three times. The presented results are the average of the three repetitions. For each test, the deformation rate was fixed at $1.10^{-3} \mathrm{~s}^{-1}$ as specified in ISO 527 .

Fig. 3 Samples ageing

Fig. 4 Cooling liquid absorption by a neat PPS

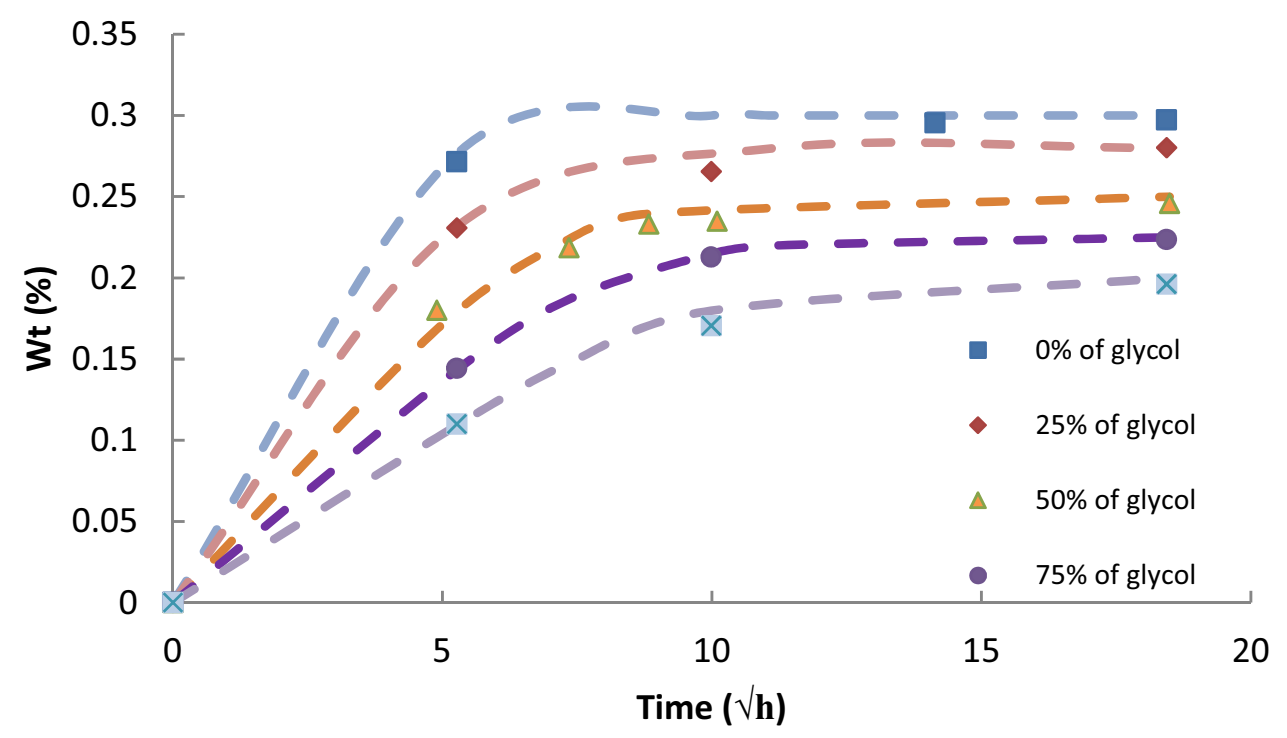

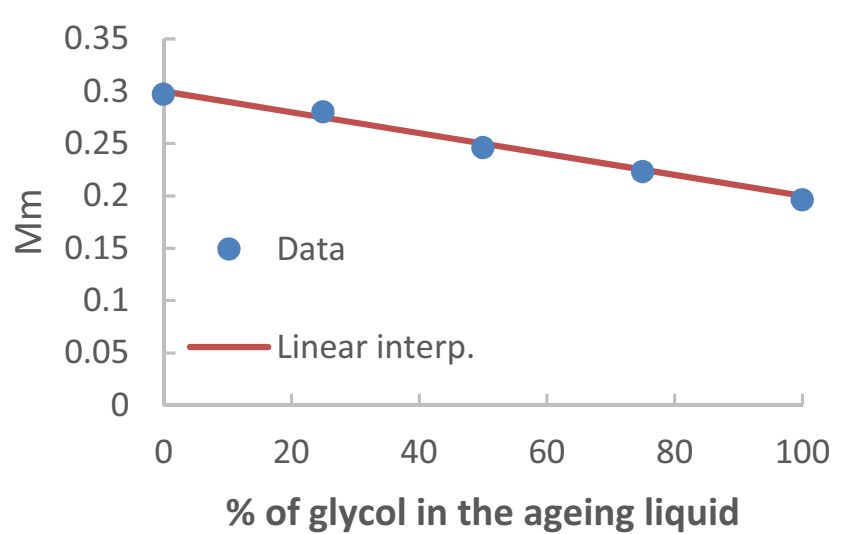

Fig. 5 Evolution of Fick's law parameters as a function of glycol proportions 


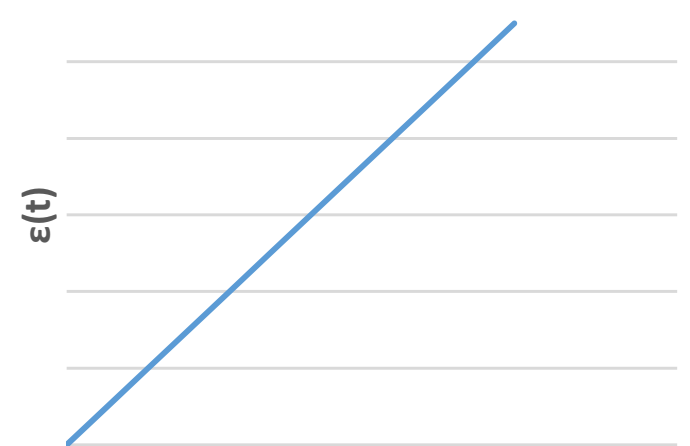

Time (s)

(a)

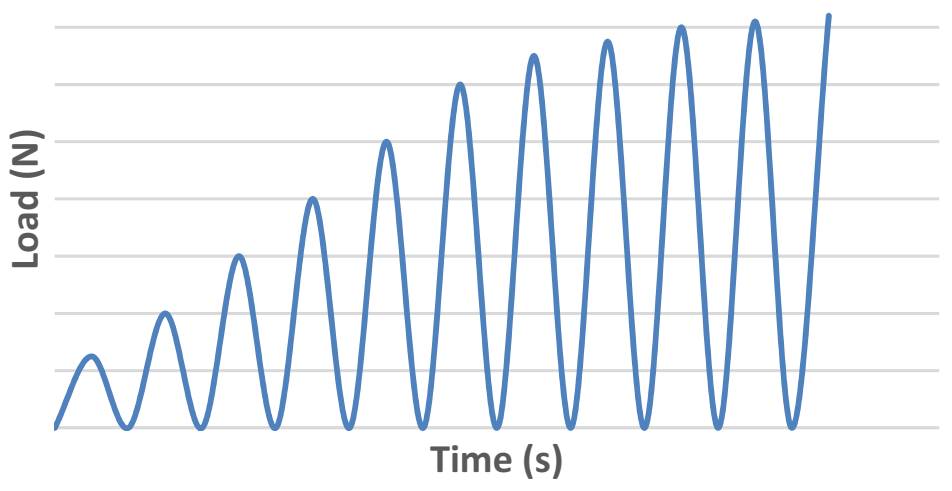

(b)

Fig. 6 a Monotonic and b cyclic mechanical tests for the experimental study

\subsection{Experimental results and mechanical analysis}

\subsubsection{Static tensile tests on the matrix}

In Fig. 7, the comparison of the mechanical behavior of aged samples for different proportions of glycol shows that the proportion of glycol has a manifest influence on the results. This can be explained by the differences in absorption, presented in Fig. 4. The mechanical behavior of the material depends on the cooling liquid absorption. For high glycol proportions, there is no significant variation in behavior, contrary to what would be expected in the event of a chemical attack.

Despite the very limited liquid absorption, hydrothermal ageing has a significant impact on the mechanical behavior of PPS. Contrary to the expected consequences for polyamide [30], a slight increase of tensile strength (about 9\%) and a clear drop in tensile strain (about $36 \%$ ) are observed with respect to DAM conditions for a standard proportion of $50 \%$ of glycol and $50 \%$ of water. With PPS, hydrothermal ageing seems to induce quite the same mechanical behavior as observed on long UVaged or thermal-aged plastics [31, 32], as the stiffness slightly increases (Fig. 8). This can be explained by the ageing process, as the stove was maintained at $90{ }^{\circ} \mathrm{C}$, inducing a physical ageing, responsible of such increase. As the aim of the glycol presence is to regulate the temperature of the liquid, the more glycol there is, the less there will be ageing effects on the mechanical behavior.

As shown in Fig. 9, the mechanical behavior of PPS is considerably influenced by the temperature, especially above the glass transition temperature $\mathrm{Tg}=90^{\circ} \mathrm{C}$. Below $\mathrm{Tg}$, the material shows an elasto-plastic type behavior. Above Tg, the behavior seems fully inelastic due to its increasing viscosity for high temperatures, especially noticeable by the appearance of a smoother increase of the stress and a delay of response linked to damping
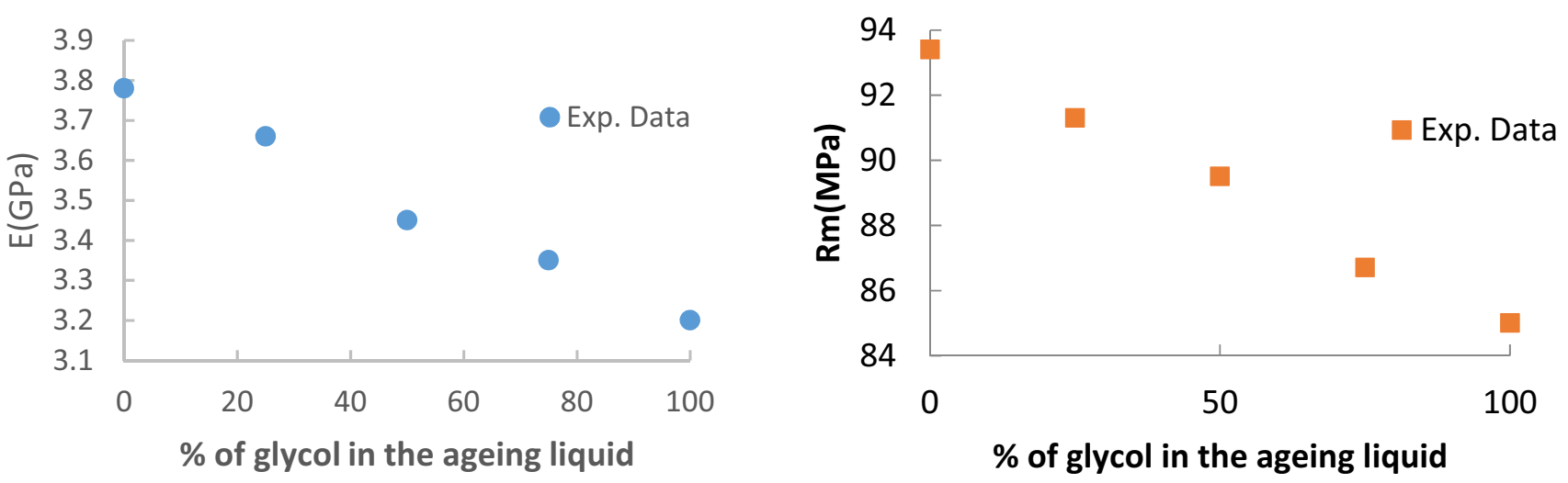

Fig. 7 Comparison of glycol proportions in the cooling liquid on the mechanical properties of a neat PPS 
Fig. 8 DAM and aged stressstrain curves comparison

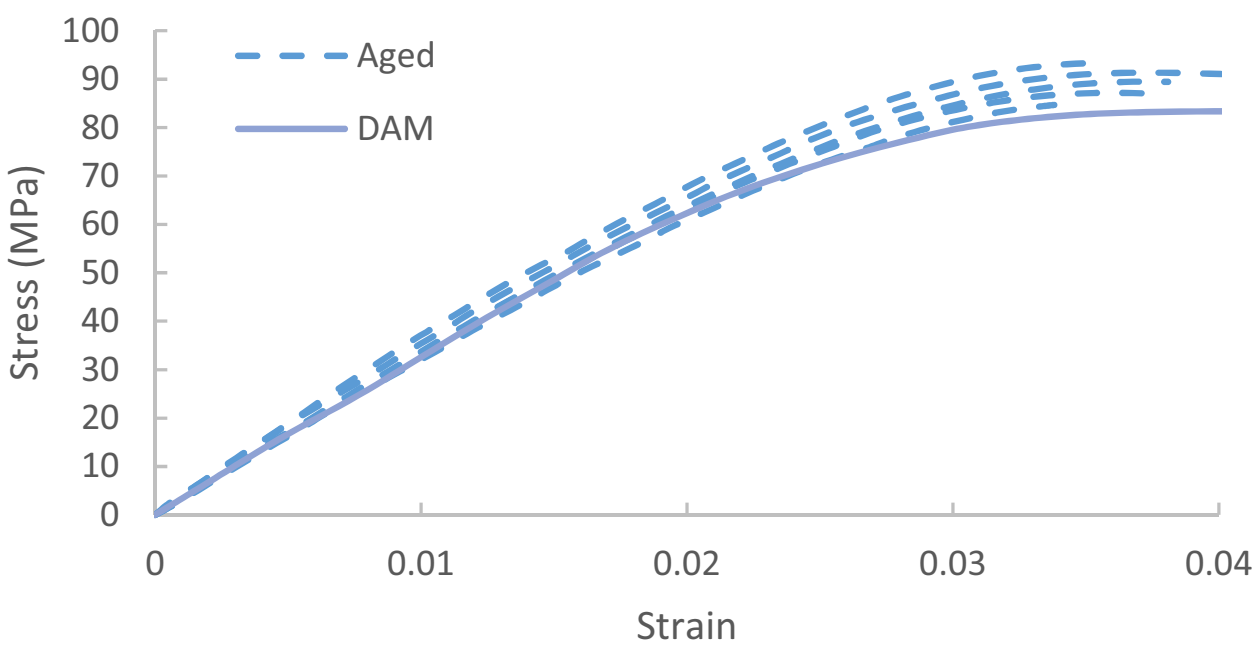

Fig. 9 Effect of temperature on tensile stress-strain curves of a neat PPS

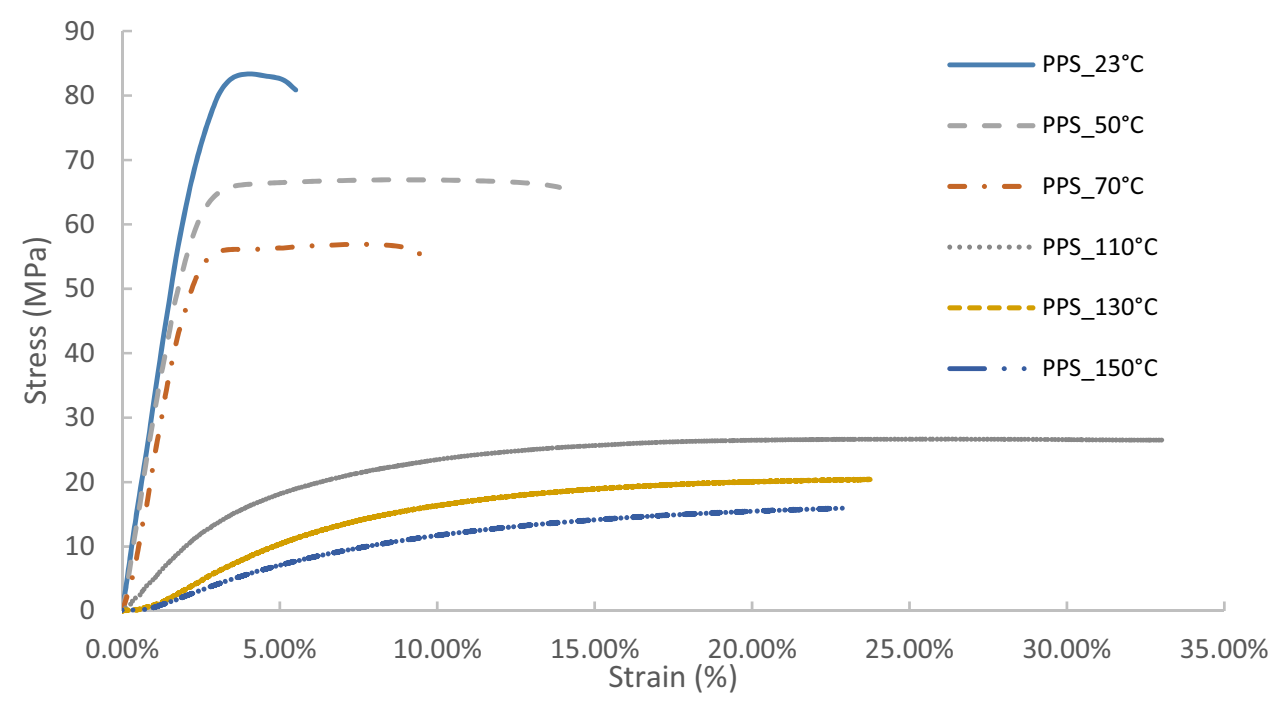

effects. This is visible at the beginning of the curve for temperatures $\mathrm{T}=130^{\circ} \mathrm{C}$ and $150^{\circ} \mathrm{C}$.

Cyclic tests were then performed in order to clearly identify the behavior through the different temperatures as well as the physical phenomena inducing these behaviors, which is essential to build a relevant and predictive model.

\subsubsection{Cyclic tensile tests on the matrix}

In the industrial application, PPS parts will mostly be used at high temperatures and will be submitted to repeated loads. Working at those temperatures means having to deal with phenomena like viscosity. The objective of those cyclic tests is then to characterize the coupled viscositydamaging evolution through the cycles and to determine the source of the modification in behavior as seen in Fig. 9, particularly once Tg is reached. Those cyclic tests are load-controlled and load-rate-controlled in order to remain in the context of the previous monotonic tensile tests, as shown in Fig. 10.

Results shown in Fig. 10 highlight two characteristic behaviors that appear under and above Tg respectively. The accumulated residual displacement at $\mathrm{T}=110{ }^{\circ} \mathrm{C}$ is about four times greater than the one observed at $\mathrm{T}=23{ }^{\circ} \mathrm{C}$. This suggests that, above $\mathrm{Tg}$, the mechanical behavior is mostly inelastic and should therefore be called "viscoplastic". In each cyclic test, the more the load rate decreases, the more the area of the hysteretic loop increases which could be due to short-term viscosity or even damage propagation. Those coupled effects lead to a cyclic softening, appearing sooner and more pronounced as the temperature increases. The slope of the bisectrix of each loop determines the computed apparent stiffness.

The previous hypothesis according to which the behavior is fully "visco-inelastic" above Tg is well 

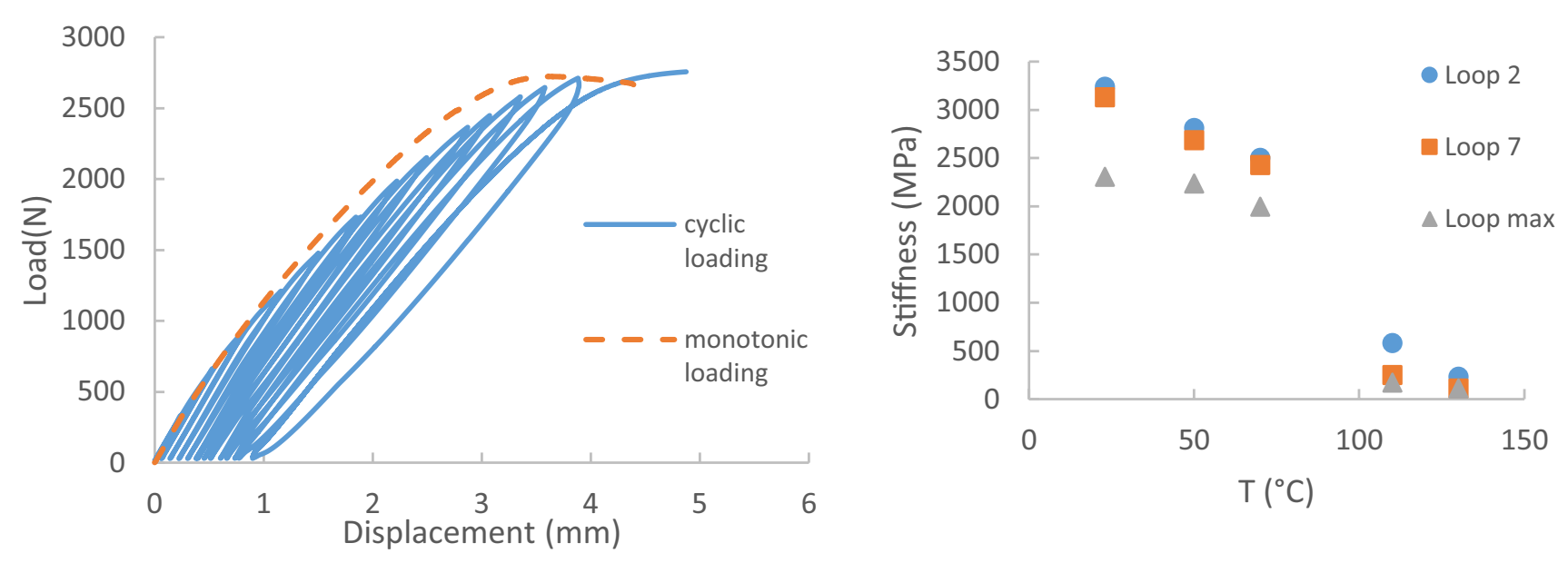

Fig. 10 Evolution of stiffness along number of loops and function of temperature

represented by the difference of magnitude in the apparent stiffness between tensile tests under and above Tg. For temperatures of $110^{\circ} \mathrm{C}$ and $130^{\circ} \mathrm{C}$, the apparent stiffness is very low, even at the first loop, which means that for these temperatures, there is no pure elastic behavior.

The cyclic softening could also be partially caused by other phenomena like self-heating. SEM could help with assessing the main origin of this cyclic softening.

The adjunction of fibers can modify the mechanical behavior as the reinforcement can reduce the sensitivity to some phenomena like viscosity. This evolution can be observed through the tensile tests performed on the composite samples with different fiber orientations.

\subsubsection{Monotonic tensile tests on composite}

In order to evaluate the modification of the mechanical behavior caused by the presence of fibers, monotonic tensile tests were performed in similar conditions as those imposed for the neat PPS. As for the characterization of the composite, it is required to take into account the fiber orientation within the sample. As shown in Fig. 11, the more the fibers are aligned with the loading direction, the more effective the reinforcement is.

The influence of temperature was studied for a fiber orientation of $0^{\circ}, 45^{\circ}$ and $90^{\circ}$ from the loading direction, as it was done for the analysis of the ageing sensitivity. The results are shown in Fig. 12.

The glass transition temperature remains clearly identifiable, whatever the fiber orientation. In addition, for a
Fig. 11 Tensile stress-strain curve for a PPS GF40 at room temperature

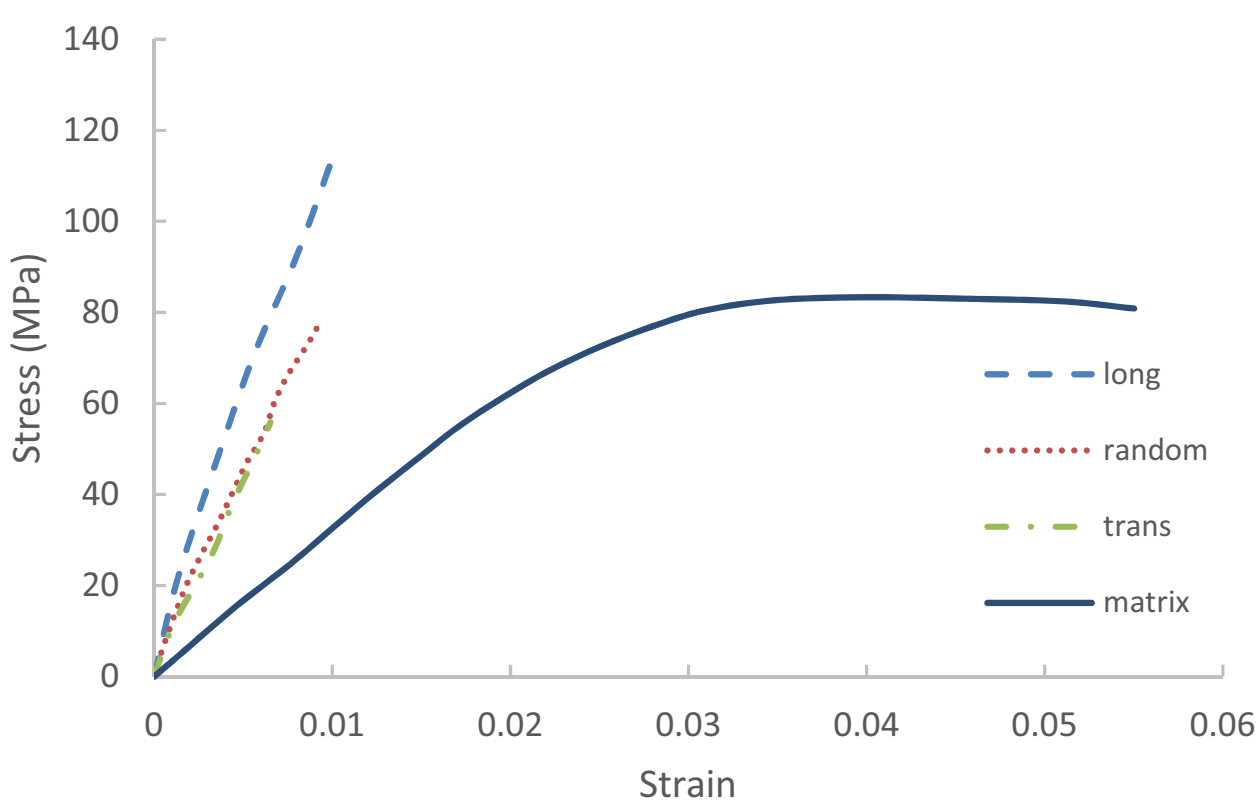


Fig. 12 Evolution of the tensile tests results through temperatures for the flow direction, a fiber orientation of $45^{\circ}$ and the cross flow direction

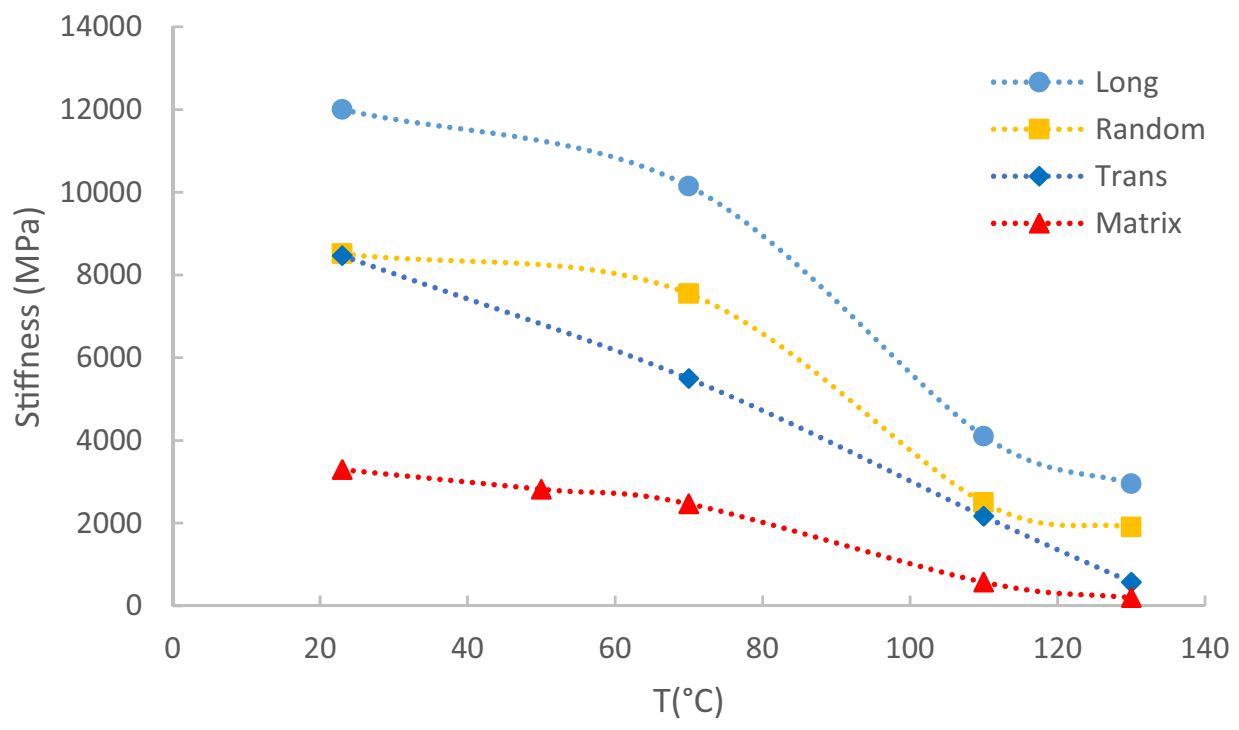

Table 2 Comparison of tensile strength evolutions for different fiber orientations

\begin{tabular}{lllll}
\hline Material & Matrix & Trans & Random & Long \\
\hline$\frac{R_{m}^{22^{\circ} \mathrm{C}}}{\boldsymbol{R}_{m}^{130^{\circ} \mathrm{C}}}$ & 4.92 & 1.79 & 2.03 & 2.35 \\
\hline
\end{tabular}

transverse fiber orientation from the loading direction, the reinforcement effect is less pronounced at high temperatures, as the matrix becomes much more distorted due to viscosity. In that case, the stiffness of the composite will reach the stiffness of the matrix faster as the fibers will not be able to compensate for the strain of the matrix.

Contrary to the neat PPS, the composite keeps a reasonable strain, even at high temperatures. It is worth noting that the fiber orientation has no major impact on the decrease of the tensile strength for any temperature, has shown in Table 2.

The results at a temperature of $130^{\circ} \mathrm{C}$ present a substantial evolution depending on the fiber orientation. The less the fibers are aligned with the loading direction, the more the damping effect is important at the beginning of the stress-strain curve. This phenomenon linked to viscosity was already observed for a neat PPS, which means that the behavior of the composite is similar to that of the matrix regarding the sensitivity to the temperature when the fibers are aligned transversally.

In order to predict the behavior of the composite at high temperatures, it is essential to build relevant and predictive constitutive equations for the matrix, as the composite behaves in the same way as the matrix once the temperature is elevated.

\section{Modelling}

\subsection{Thermomechanical framework}

\subsubsection{Rheological model}

The objective of this section is to present the formulation of a thermodynamics-based phenomenological model including the physical phenomena described in Sect. 2. As these data were obtained by means of tensile tests, the uniaxial solicitation can be described by a 1D-rheological scheme, given in Fig. 13. This kind of rheological scheme is widely used in polymer and composite modelling [23, 26, 33]. The following equations have been formulated within the mechanical framework of Lemaitre and Chaboche [34], and have been used in many other recent studies $[26,35,36]$.

The rheological model consists of the following elements:

- One single linear spring

- $\mathrm{N}$ viscoelastic Kelvin-Voigt branches constituted of a linear spring and a linear dashpot mounted in parallel to define short-term and long-term viscoelastic strains. The consideration of more than two KelvinVoigt branches allows for creep and relaxation effects to be taken into account in a wide range of loading frequencies [27].

- One viscoplastic branch, constituted of a non-linear spring and a non-linear dashpot, mounted in parallel.

The mounting in series of all those elements leads to the following total strain: 


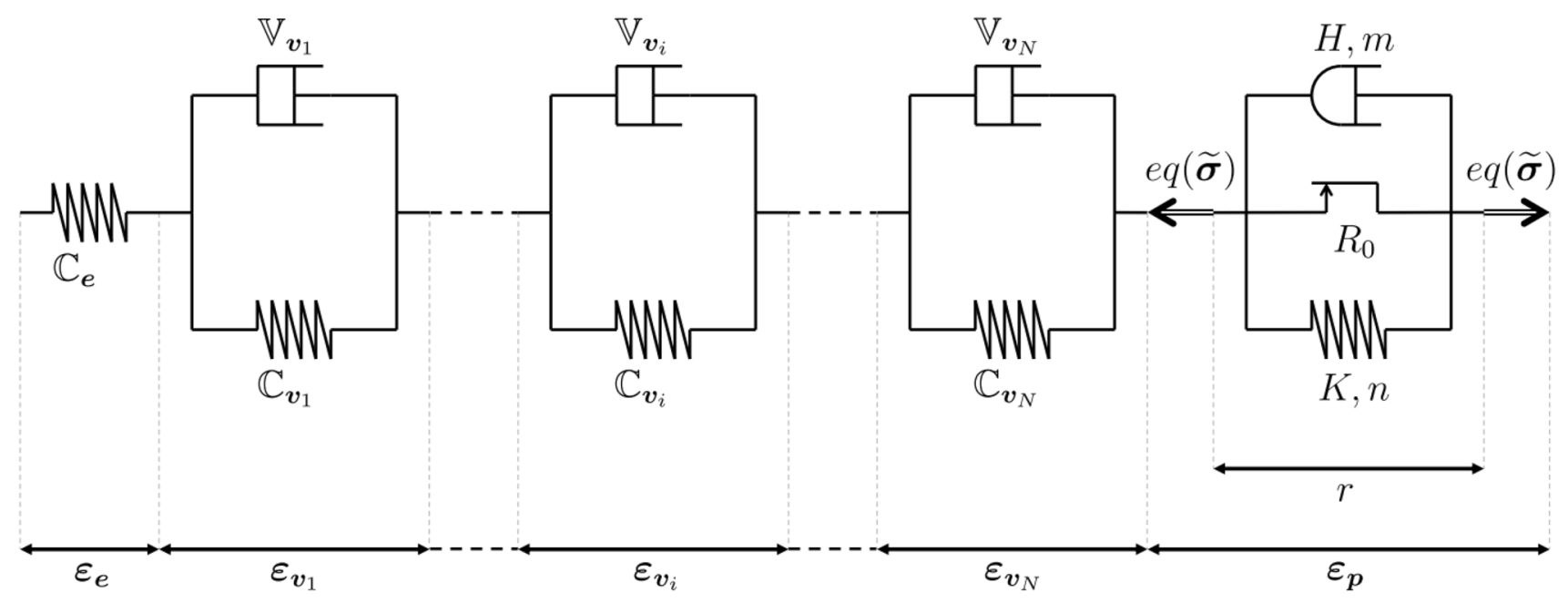

Fig. 13 1D-rheological scheme of the proposed constitutive model

$\varepsilon=\varepsilon_{e}+\sum_{i=1}^{N} \varepsilon_{v i}+\varepsilon_{v p}$

For the sake of simplicity in the following equations, the Einstein summation convention was adopted, leading to the following form:

$\varepsilon=\varepsilon_{e}+\varepsilon_{v i}+\varepsilon_{v p}$

A Helmholtz free energy expression can thus be written as the sum of stored energies:

$$
\begin{aligned}
\rho \psi= & {\left[\frac{1}{2} C_{e}\left(\varepsilon-\varepsilon_{v i}-\alpha \theta\right)^{2}+\frac{1}{2} C_{v i} \varepsilon_{v i}^{2}\right] e^{\delta_{e}\left(1-\frac{T_{e q}}{T_{0}}\right)}-\frac{\rho C}{2 T_{0}} \theta^{2} } \\
& -C_{v i} \varepsilon_{v i} \alpha \theta+\left[\frac{1}{2} k p^{2}+R_{\infty}\left(p+\frac{1}{m} e^{-m p}\right)\right] e^{\delta_{p}\left(1-\frac{T_{e q}}{T_{0}}\right)}
\end{aligned}
$$

In order to define the mechanical state at any temperature, as well as the associated thermodynamic forces, the Helmholtz free energy expression is defined by several scalar-state variables and parameters:

- The overall strain $\varepsilon$, an outside control variable, directly associated to $\sigma$

- The viscoelastic strains $\varepsilon_{v i}$, including the short-term and long-term viscoelastic strains and more if needed

- The pseudo-viscoplastic or "visco-inelastic" strain $\varepsilon_{v p}$

- The hardening exponent $m$

- The hardening modulus $R_{\infty}$ in $\mathrm{MPa}$

- The linear-hardening variable $k$ in $\mathrm{MPa}$

- An Arrhenius-type variable $\delta_{e}$, allowing the prediction of elastic behavior through temperature

- An Arrhenius-type variable $\delta_{p}$, allowing the prediction of pseudo-viscoplastic behavior through temperature
- The coefficient of linear thermal expansion a

- The mass thermal capacity $C$

The particularity of the presented model is the introduction of the Arrhenius-type coefficients, as they were used in other studies in order to evaluate temperature sensitivity [37, 38]. As the present model is designed to take into account the variation of temperature, the latter is then defined as a variable $\theta$, the difference between the current temperature $T$ and the reference temperature $T_{0}$ :

$\theta=T-T_{0}$

For the numerical application of the law, we chose to work with $p$, the cumulative viscoplastic strain and $\varepsilon_{e}$, the elastic strain, which is not an internal variable but is convenient for the equation writing and for the characterization on a stress-strain curve.

The combination of the first and second laws of thermodynamics can be expressed by the Clausius-Duhem inequality [39]:

$D=\sigma \dot{\varepsilon}-\rho(\dot{\psi}+s \dot{\theta})-\frac{1}{T_{0}} \operatorname{qgrad}_{x} T \geq 0$

where $q$ is the heat flux, $\rho$ the mass density and $s$ the entropy, the latter always being non-negative.

In order to describe the evolution of internal variables and the viscoelastic dissipation process, a pseudo-dissipation potential is defined:

$\varphi\left(\dot{\varepsilon}_{v i}\right)=\frac{\tau\left(C_{e}+C_{v i}\right)}{2} \dot{\varepsilon}_{v i}^{2}$

where $\tau$ is the stress relaxation time. 
The state equations of the problem can then be deduced by differentiation of the potential with respect to the state variables:

$\sigma=\rho \frac{\partial \psi}{\partial \varepsilon}$

$s=-\frac{\partial \psi}{\partial \theta}$

$\rho \frac{\partial \psi}{\partial \varepsilon_{v i}}=-\frac{\partial \varphi\left(\dot{\varepsilon}_{v i}\right)}{\partial \dot{\varepsilon}_{v i}}$

$R(p)=\rho \frac{\partial \psi}{\partial p}$

These constitutive relations lead to the equations allowing the modelling of stress-strain curves, for viscoelastic parts and pseudo-viscoplastic parts, defined by Eqs. (12a) and (14a):

$\sigma(\varepsilon)=\frac{C_{e} C_{v i}}{C_{e}+C_{v i}}\left[(\varepsilon-\alpha \theta) e^{\delta_{e}\left(1-\frac{T}{T_{0}}\right)}-\alpha \theta\right]\left[1-e^{-\frac{\varepsilon^{\delta_{e}}\left(1-\frac{T}{T_{0}}\right)}{\dot{\varepsilon} \tau}}\right]$

With a yield stress that is also temperature dependent:

$\sigma_{y}(T)=\sigma_{y}^{T_{0}} e^{\delta_{e}\left(1-\frac{T}{T_{0}}\right)}$

$R(p)=\left[k p+R_{\infty}\left(1-e^{-m p}\right)\right] e^{\delta_{p}\left(1-\frac{T}{T_{0}}\right)}$

And the following yield function, corresponding to the well-known von Mises surface $f$ :

$f(\sigma, R, T)=\sigma_{e q}-\sigma_{y}(T)-R(p)$

Those state laws define the behavior of the material under Tg. As it was previously suspected in Sect. 1 and observed in Sect. 2, the behavior above Tg is completely different. Indeed, it was shown in Sect. 2 that at high temperatures, the mechanical behavior of the PPS is fully pseudo-viscoplastic. In order to express that phenomenon, only a revised version of Eq. (14a) is used for temperatures above the glass transition:

$R(p)=\left[k p+R_{\infty}\left(1-e^{-m p}\right)\right]\left[1-e^{-\frac{p e^{\delta\left(1-\frac{T}{T_{0}}\right)}}{\dot{\varepsilon} \tau}}\right] e^{\delta\left(1-\frac{T}{T_{0}}\right)}$

These equations describe the mechanical behavior within a wide range of temperatures, under and above the glass transition. As shown in Sect. 2, hydrothermal ageing has similar effects as the variation of temperature on the mechanical behavior. It is then possible to consider the ageing process in the model.

\subsubsection{Temperature-humidity equivalence}

As it was shown by [23] for polyamides, humidity affects the glass transition temperature. Therefore, it is really important to be able to estimate the current glass transition temperature to know which model is adapted. In order to take into account this variation, the presented model has to be adapted. Since the glass transition temperature does not appear explicitly in the model, a redefinition of the concept of temperature is needed.

The important parameter that reports the mechanical behavior of a material depending on the temperature is the deviation from the glass transition temperature. Thus, if $\mathrm{Tg}$ decreases, the difference in temperature increases. It is possible to increase this gap by passing on this glass temperature modification on the loading temperature.

The equivalent current temperature will then depend on the percentage of humidity, and can be defined, with respect to the equation of [23] and with respect to the experimental results shown in Sect. 2 , by the following equation:

$T_{e q}=T+\chi H(\%)$

where $H(\%)$ is the percentage of humidity and $\chi$ is the slope.

In our applications, the material is always fully immersed. Moreover, the ambient liquid is composed of water and glycol. In order to evaluate the effect of each part of the cooling liquid, the Eq. (17a) was upgraded as follows:

$T_{\text {eq }}=T-(\chi H(\%)+\xi G(\%))$

where $G(\%)$ is the glycol proportion in the liquid, $H(\%)$ is the water proportion and $\xi$ and $\chi$ are the slopes.

In order to consider the industrial application and the worst-case scenario for the mechanical behavior, the study was only conducted with a material fully immerged and tested at saturation.

The model can be completed to take into account hydrothermal effects by introducing Eq. (17b):

- Hydro-thermo-viscoelasto-pseudo-plastic before Tg:

$$
\begin{aligned}
& \sigma(\varepsilon)=\frac{C_{e} C_{v i}}{C_{e}+C_{v i}}\left[(\varepsilon-\alpha \theta) e^{\delta_{e}\left(1-\frac{T_{e q}}{T_{0}}\right)}-\alpha \theta\right]\left[1-e^{-\frac{\varepsilon e_{e}\left(1-\frac{T_{e q}}{T_{0}}\right)}{\dot{\varepsilon} \tau}}\right] \\
& R(p)=\left[k p+R_{\infty}\left(1-e^{-m p}\right)\right] e^{\delta_{p}\left(1-\frac{T_{e q}}{T_{0}}\right)}
\end{aligned}
$$


- Hydro-thermo-viscoelasto-pseudo-plastic after Tg:

$$
R(p)=\left[k p+R_{\infty}\left(1-e^{-m p}\right)\right]\left[1-e^{-\frac{p e^{\delta\left(1-\frac{T_{e q}}{T_{0}}\right)}}{\varepsilon \tau}}\right] e^{\delta\left(1-\frac{T_{e q}}{T_{0}}\right)}
$$

\subsection{Application}

\subsubsection{Parameters identification and correlation}

As previously presented, for clarity reasons, $\varepsilon_{e}$ is used. Equation $12 \mathrm{~b}$ can be rewritten as follows:

$\sigma(\varepsilon)=\frac{C_{e} C_{v i}}{C_{e}+C_{v i}}\left[\varepsilon_{e} e^{\delta_{e}\left(1-\frac{T_{e q}}{T_{0}}\right)}\right]\left[1-e^{-\frac{\delta_{e}\left(1-\frac{T_{e q}}{T_{0}}\right)}{\varepsilon \tau_{i}}}\right]$

The first step is the modelling of the stress-strain curve obtained at the reference temperature $\mathrm{T}_{0}$. The mechanical parameters can then be found, including $\sigma_{y}^{T_{0}}$, by a leastsquares fitting method. Once the mechanical parameters are obtained, the second step is to evaluate the thermal shift parameters $\delta_{e}$ and $\delta_{p}$, which express the impact of temperature on the mechanical properties. To do so, the previous parameters are fixed and the thermal parameters can be determined from the shift the stress-strain curve obtained at a different temperature. Once those parameters are known, this protocol is repeated in order to evaluate the influence of the glycol proportion in the cooling liquid on the mechanical properties. The parameters $\chi$

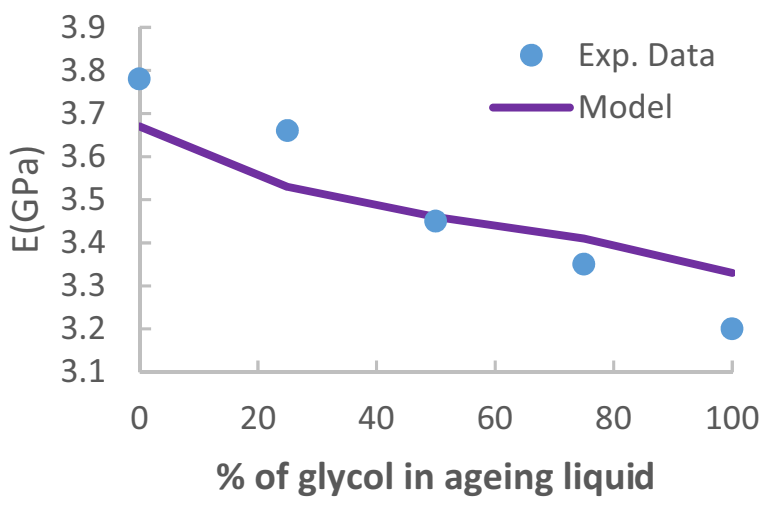

(a) and $\xi$ can respectively be isolated on the tensile curves obtained for a full water ageing and for a full glycol ageing. The curves corresponding to a proportion of $25 \%, 50 \%$ and $75 \%$ of glycol respectively are thus fully predicted.

As the parameters are fully fixed, the influence of the immersion and glycol proportions can be quantified for the industrial application, and illustrated by Fig. 14 .

This means that the immersion of the PPS in a coolant liquid composed of $50 \%$ of water and $50 \%$ of glycol for $400 \mathrm{~h}$ is equivalent to testing the DAM material at a lower temperature. From the industrial point of view, this kind of results is interesting because, for immerged under-thehood components, the effects of absorption can be represented in the form of a temperature difference. It can be of interest for carmakers and automotive component manufacturers to build material libraries with the characteristic equivalent temperature of the immerged state.

All of the material's parameters necessary to characterize and predict the mechanical behavior of the neat PPS are listed in Table 3. In order to be more explicit and to facilitate the understanding, $\frac{C_{e} C_{v i}}{C_{e}+C_{v i}}$ is presented as the equivalent apparent modulus $E$, in MPa.

The same methodology was used in order to obtain the parameters of the model above the glass transition temperature. A new reference temperature has to be fixed, not too close to the glass transition temperature if possible, in order to avoid any transition effect between the two states of the material.

In the same way, the mechanical parameters are evaluated on the curve obtained at the new reference

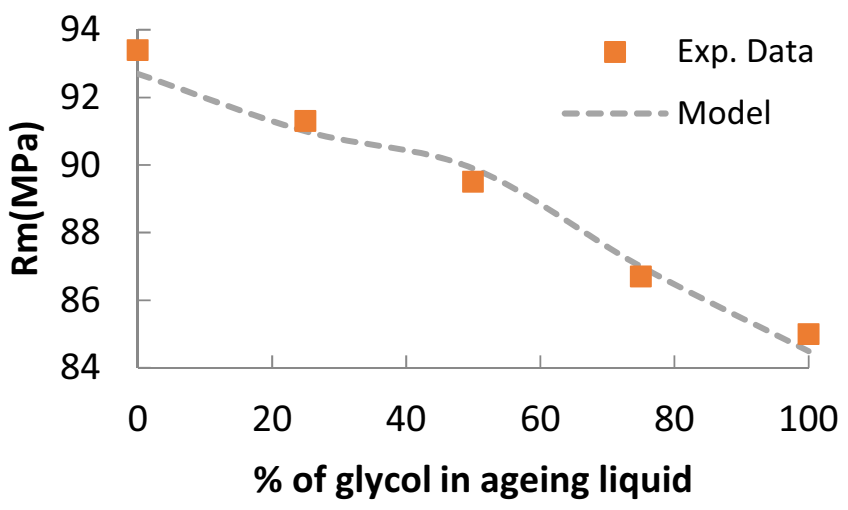

(b)

Fig. 14 Modelling of stress-strain curves of the aged PPS

Table 3 Material parameters for a neat PPS under Tg

SN Applied Sciences

\begin{tabular}{lllllllll}
\hline $\mathrm{E}(\mathrm{MPa})$ & $\mathrm{k}(\mathrm{MPa})$ & $\mathrm{R}(\mathrm{MPa})$ & $\mathrm{m}$ & $\delta \mathrm{e}$ & $\delta \mathrm{p}$ & $\mathrm{T}(\mathrm{s})$ & $\chi(\mathrm{K})$ & $\xi(\mathrm{K})$ \\
\hline 3216.48 & 0.00 & 86.70 & 73.66 & 1.73 & 2.43 & 2.34 & 16.99 & 6.62 \\
\hline
\end{tabular}


temperature, above Tg. The modelled curve also represents the basis of future predictions. The next step was the determination of the Arrhenius shift factor $\delta$, once again with a least-squares fitting method on a stress-strain curve obtained at a higher temperature.

The modelling of the mechanical behavior of the PPS for a temperate higher than $\mathrm{Tg}$ can thus be performed with the parameters listed in Table 4.

Figure 15 shows that the reduced number of parameters presented in Tables 3 and 4 obtained with fittings at $23^{\circ} \mathrm{C}, 70^{\circ} \mathrm{C}, 110^{\circ} \mathrm{C}$ and $130^{\circ} \mathrm{C}$ respectively are sufficient to predict complete tensile curves for the elastic and viscoplastic parts at other temperatures under and above glass transition, taking into account viscosity effects like damping.

As shown in Fig. 16, the proposed model only needs two stress-strain curves per part (under and above $\mathrm{Tg}$ ), and two others to consider the ageing effects in a twoconstituent liquid and to define the equivalent temperatures in order to predict the mechanical properties for a wide range of environmental configurations.

The proposed model presents the evolution of the mechanical behavior of the material regarding its loading environment, and is able to predict said behavior. Nevertheless, even if the constitutive model is relevant for the industrial application, it may not be a sufficient approach for the entire range of experimental conditions, especially for uploading-unloading tests combining immersion and temperature [40].
In order to demonstrate the accuracy of these constitutive equations, this model was confronted to another model so as to evaluate the possible room for improvement regarding what can be considered the maximal possible accuracy.

\subsubsection{Comparison with a sequential model}

In order to evaluate our possible improvement, a sequential artificial-intelligence-based model was built to fit the experimental curves. Artificial intelligence is already used in polymer physics [41, 42]. Artificial intelligence algorithms are used in many fields to help making predictions from stochastic data. One of the strengths of this method is its ability to build a model by training on those data with a given number of iterations, by means of a repetitive loss reduction algorithm. Hence, the Al-based constructed model is not a rigorous mathematical model contrary to a mathematical demonstration, but this method enables the convergence of some difficult calculations and fittings with an extreme precision, providing there are enough data and iterations.

From a conceptual point of view, this means that we can reach a better accuracy with $\mathrm{Al}$ than with mathematical models, because $\mathrm{Al}$ is not subject to the physical reality of the parameters. Thus, even if the training model can be improved, it can easily be seen that no rational proposed model, pre-existing or not, can present a lower average error than the one presented by an artificial intelligence model.

Table 4 Material parameters for a neat PPS above Tg

\begin{tabular}{lllllll}
\hline $\mathrm{E}(\mathrm{MPa})$ & $\mathrm{k}(\mathrm{MPa})$ & $\mathrm{R}(\mathrm{MPa})$ & $\mathrm{m}$ & $\delta \mathrm{e}$ & $\delta$ & $\mathrm{T}(\mathrm{s})$ \\
\hline & 3.58 & 26.52 & 24.63 & & 5.17 & 26.64 \\
\hline
\end{tabular}

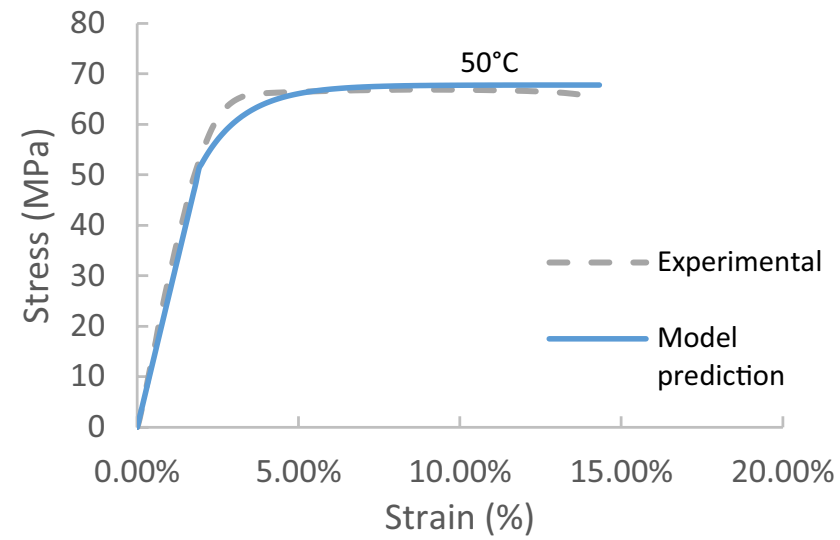

(a)

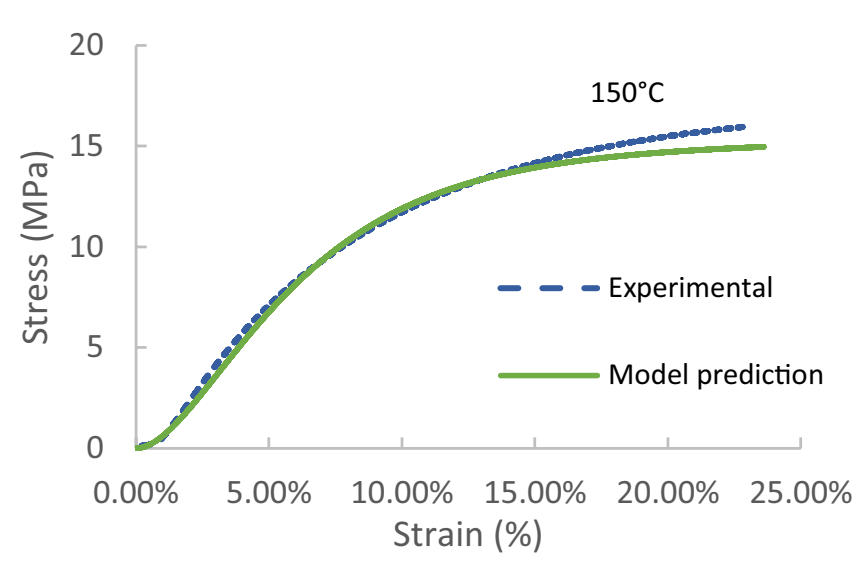

(b)

Fig. 15 Tensile curves predictions under $\mathbf{a}$ and above $\mathbf{b}$ glass transition temperature 
Fig. 16 Global correlation depending on the temperature

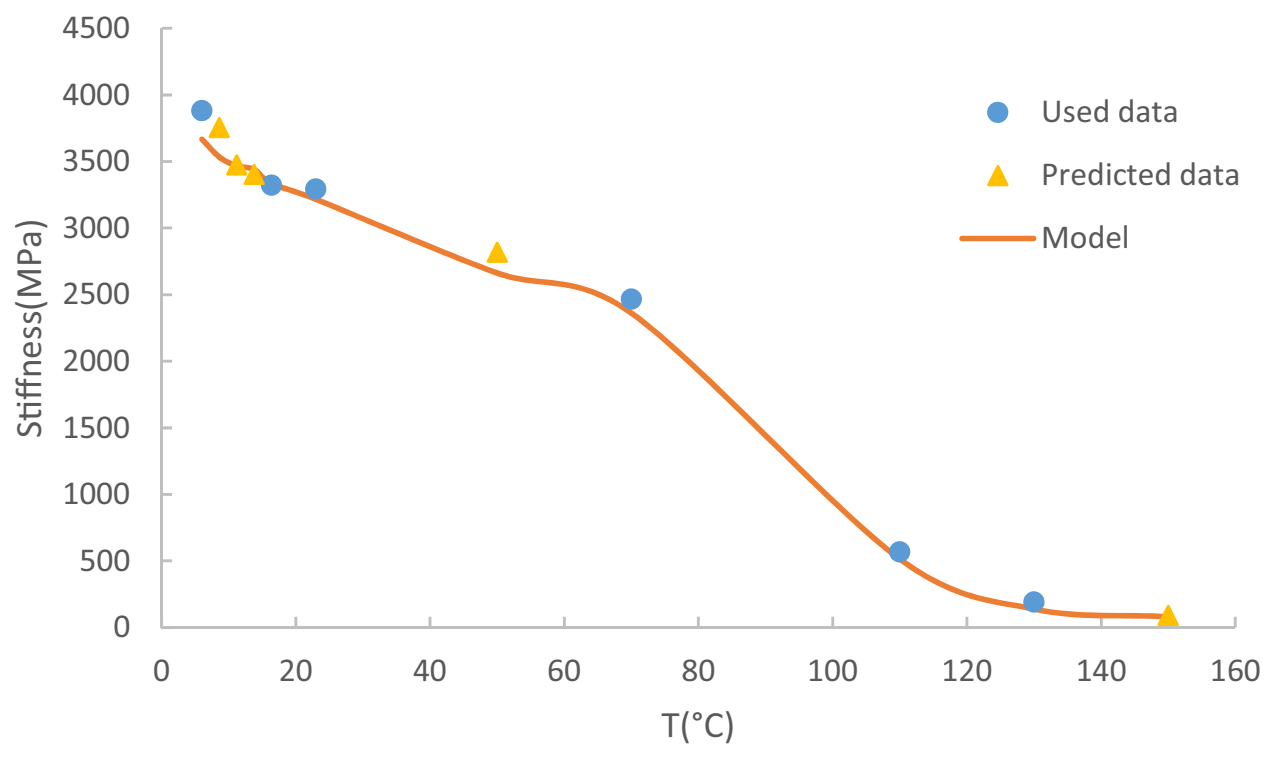

The aim of the fitting of the tensile curves by a sequential model is to show the room for improvement of our model, compared to the maximal reachable accuracy, given by the artificial intelligence results.

In order to work in the same conditions for all the tensile curves, approximately the same amount of data was used to feed the sequential model. The number of epochs was fixed at 10,000, allowing the stabilization of the error, and the model was trained on 5000 couples of input and output data points.

A global comparison of the average error of the models is then possible, as presented in Table 5.

Even if the presented model can be considered accurate, the precision could be increased by improving the reliability of the temperature control system. Indeed, the temperature used in the model is the temperature in the climatic chamber. Thus, a variation of one or two degrees Celsius between the climatic chamber and the real temperature in the core of the sample is possible. Also, the phenomenological nature of the presented model can error-prone. In order to reach a better accuracy, micro parameters are needed, such as molecular chain motion, which is induced by the high temperatures. However, quantifying this phenomenon can be very difficult, all the more in an industrial context, placing these kind of parameters beyond the scope of the study. Both models are showing different overall errors for the different configurations. This can be explained by the shape of the curve which can be harder to model for the sequential model, especially when damping effects occur.

The results given by the sequential model being used as the reference, it is worth noticing that for the majority of studied temperatures, our room for improvement is only
Table 5 Comparison of the models' accuracy

\begin{tabular}{|c|c|c|}
\hline \multirow[t]{2}{*}{$\mathrm{T}$} & \multicolumn{2}{|l|}{ Overall error } \\
\hline & $\begin{array}{l}\text { Proposed model } \\
\text { (\%) }\end{array}$ & $\begin{array}{l}\text { Sequential } \\
\text { model (\%) }\end{array}$ \\
\hline $23^{\circ} \mathrm{C}$ & 2.41 & 0.93 \\
\hline $50^{\circ} \mathrm{C}$ & 3.93 & 1.13 \\
\hline $70^{\circ} \mathrm{C}$ & 3.85 & 2.28 \\
\hline $110^{\circ} \mathrm{C}$ & 0.98 & 0.18 \\
\hline $130^{\circ} \mathrm{C}$ & 2.76 & 1.75 \\
\hline $150^{\circ} \mathrm{C}$ & 3.41 & 2.20 \\
\hline Aged at $0 \%$ of glycol & 2.40 & 1.30 \\
\hline Aged at $25 \%$ of glycol & 3.41 & 1.27 \\
\hline Aged at $50 \%$ of glycol & 2.07 & 0.70 \\
\hline Aged at $75 \%$ of glycol & 2.17 & 1.69 \\
\hline Aged at $100 \%$ of glycol & 1.78 & 0.93 \\
\hline
\end{tabular}

Bold-Completely known

Italic_Partially known

Bolditalic-Completely predicted

about $1 \%$ or $2 \%$ which confirms the good accuracy of the proposed model.

\section{Discussion}

\subsection{Physical analysis of the model parameters}

Studying the parameters obtained in Sect. 3 can demonstrate how the mechanical behavior changes depending on the temperature and which mechanisms are involved. The comparison of the parameters with the experimental results of Sect. 2 also offers the possibility to grasp the 
physical meaning of the model parameters. Tables 3 and 4 highlight the transition between the glassy state and the rubbery state of the neat PPS. Indeed, as presented in Sect. 2.4.2, viscosity becomes the leading factor defining the mechanical behavior as the temperature increases. The strain of the material increases, thus inducing a decrease of its stiffness. This influence is described by the parameter $\delta$. Moreover, the presence of viscosity implies the presence of damping effects, materialized by a delay of the response to a solicitation. This characteristic time is the parameter $\mathrm{t}$. In Table 3, the leading parameters are the mechanical ones, defining an elasto-plastic type behavior. However, as shown in Table 4, those previous parameters are considerably less influent above $\mathrm{Tg}$, where the parameters related to viscosity become more predominant, which is fully consistent with the results of the cyclic tests. The results of those tests and the model enable to state that, above the glass transition temperature, the mechanical behavior of the PPS is "visco-inelastic", also called "pseudo-viscoplastic".

\subsection{Impact of fibers on viscosity}

The reaction of a polymer to the temperature is due to the molecular chain motion inside the material. Hence, in a short-glass-fiber-reinforced plastic, only the matrix reacts to the temperature [43]. As shown in Sect. 4.1, each parameter of the presented model is linked to a physical phenomenon. This makes it possible to study the influence of the fiber ratio and the fiber orientation on the composite's global viscosity by analyzing the evolution of the model parameters. As it was shown in [23], this kind of phenomenological models can also be applied to composites, as long as the fiber orientation remains fixed. In our case, applying the model to the composite is only relevant for the study of the variation of the parameters, as presented in Table 6 and Fig. 17.

Contrary to the neat PPS, the PPS GF40 does not necessarily have values for $\tau$ as the presence of fibers seems to diminish this damping effect. For $\delta_{p}$, just like for the composite's stress-strain curves, and for a certain range of temperatures, there is no pseudo-plastic part. Since the model parameters are a function of the fiber orientation, their evolution can be modelled.
Table 6 Model parameters for a PPS GF40: (a) in moldflow direction, (b) with a fiber orientation of $45^{\circ}$ and (c) in cross-flow direction

\begin{tabular}{|c|c|c|c|c|c|c|c|c|c|c|c|}
\hline $\mathrm{E}(\mathrm{MPa})$ & $\mathrm{k}(\mathrm{MPa})$ & $\mathrm{R}(\mathrm{MPa})$ & $\mathrm{m}$ & Se & $\tau(s)$ & $\mathrm{E}(\mathrm{MPa})$ & $\mathrm{k}(\mathrm{MPa})$ & $\mathrm{R}(\mathrm{MPa})$ & $\mathrm{m}$ & $\delta$ & $\tau(s)$ \\
\hline \multicolumn{6}{|c|}{ (a) Longitudinal before $\mathrm{Tg}$} & \multicolumn{6}{|c|}{ Longitudinal after $\mathrm{Tg}$} \\
\hline $11,781.65$ & 0 & 165.019 & 76.34 & 0.61 & & & 0 & 63.95 & 87.29 & 0.52 & 1.69 \\
\hline \multicolumn{6}{|c|}{ (b) Random before $\mathrm{Tg}$} & \multicolumn{6}{|c|}{ Random after Tg } \\
\hline 8721.99 & 0 & 103.37 & 104.91 & 0.91 & & & 0 & 51.44 & 68.057 & 4.85 & 1.97 \\
\hline \multicolumn{6}{|c|}{ (c) Transversal before $\mathrm{Tg}$} & \multicolumn{6}{|c|}{ Transversal after Tg } \\
\hline 8277.97 & 0 & 106.39 & 79.019 & 1.67 & 0.56 & & 0 & 50.13 & 69.47 & 5.95 & 6.24 \\
\hline
\end{tabular}

Fig. 17 Model prediction of the stiffness evolution through temperature with different fiber orientations

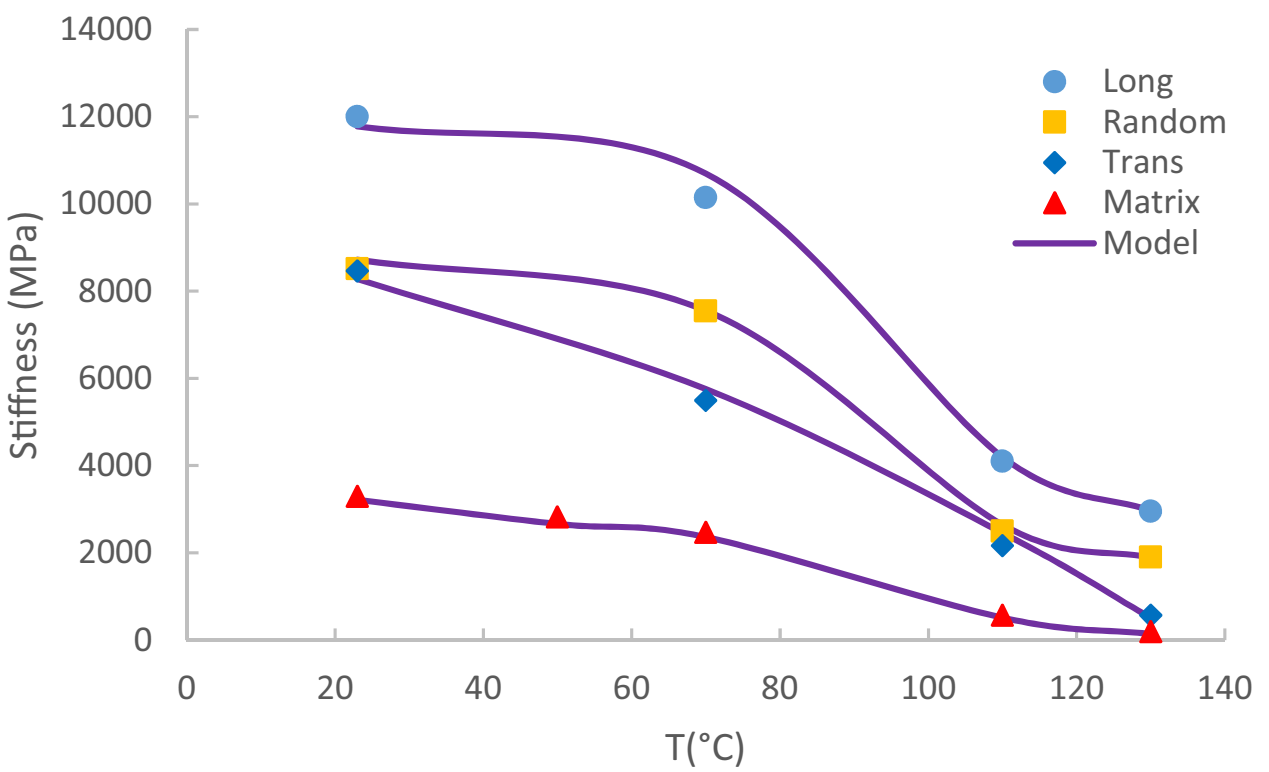

SN Applied Sciences 
Figure 18 displays the evolution of $\delta$ as a function of the fiber orientation, with the following normalized notation: 0 represents the cross flow direction and 1 represents the flow direction. Under Tg, there is no significant pseudoplastic behavior of the composite; that is why only $\delta_{\mathrm{e}}$ is presented for this range of temperatures. Moreover, data obtained for the matrix are also displayed in Fig. 18 to study the difference between the value for the matrix and the value obtained at cross-flow direction. Indeed, it was shown in [30] that a composite with fibers mostly oriented in the cross-flow direction has a similar behaviour to that of the matrix. The possible difference between the crossflow and matrix parameters can then be attributed to the fiber ratio, all the more in this case because the studied composite is highly reinforced, as the glass does not present a viscous behavior at any temperature.
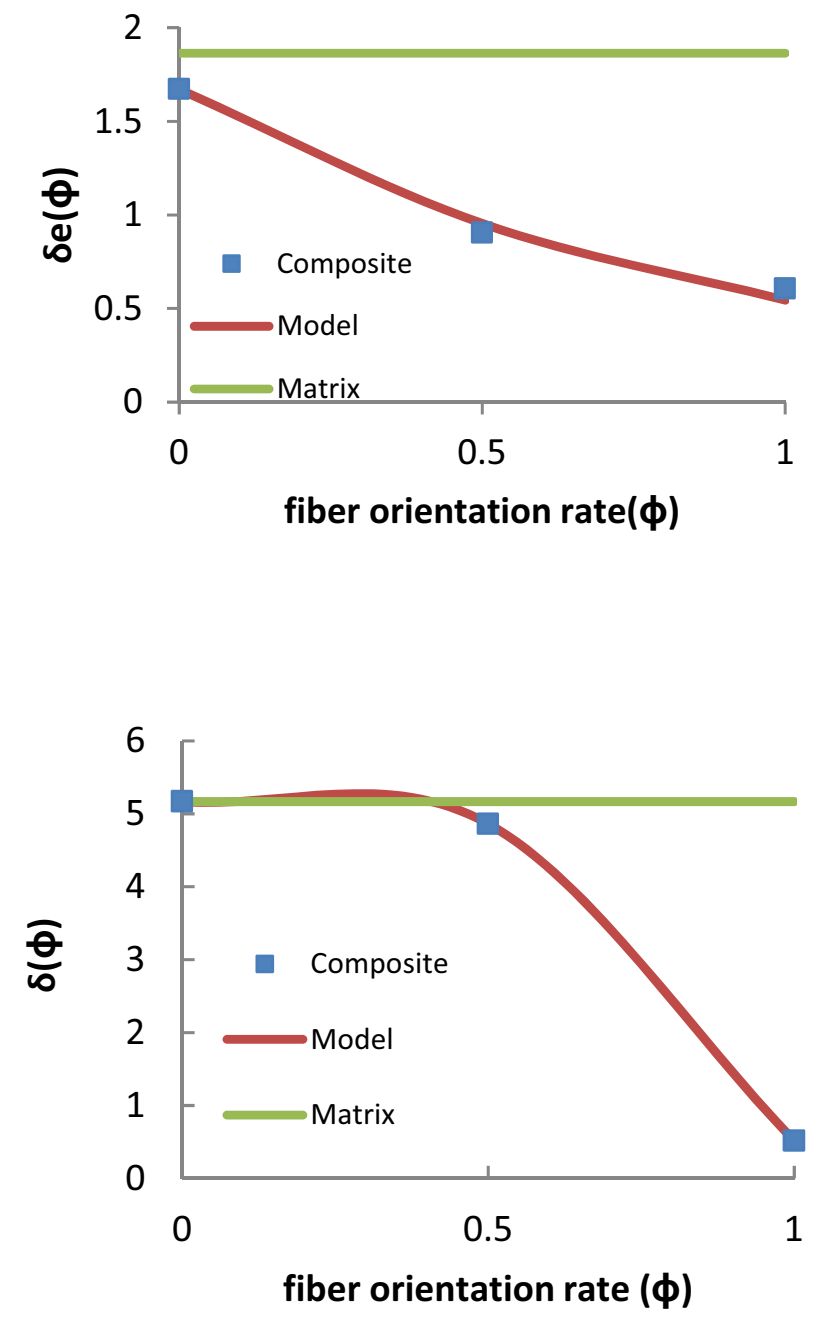

It is worth noting that the dynamics of evolution under and above $\mathrm{Tg}$ are completely different. In both cases, the more the fibers are aligned with the loading direction, the less the strength is impacted by the temperature, i.e. the loss of properties induced by the viscosity is sensibly stymied. However, above $\mathrm{Tg}$, the fibers have to be aligned with the loading direction for the fiber orientation to have more consequences on the material's strength, whereas under Tg, even if the fibers are not completely aligned with the loading direction, the fiber orientation has a significant impact on the material's strength. Additionally, the stress relaxation time does not show any different dynamic variation for the entire range of temperatures.

A phenomenological law can thus be proposed to quantify the different evolutions, based on the matrix:

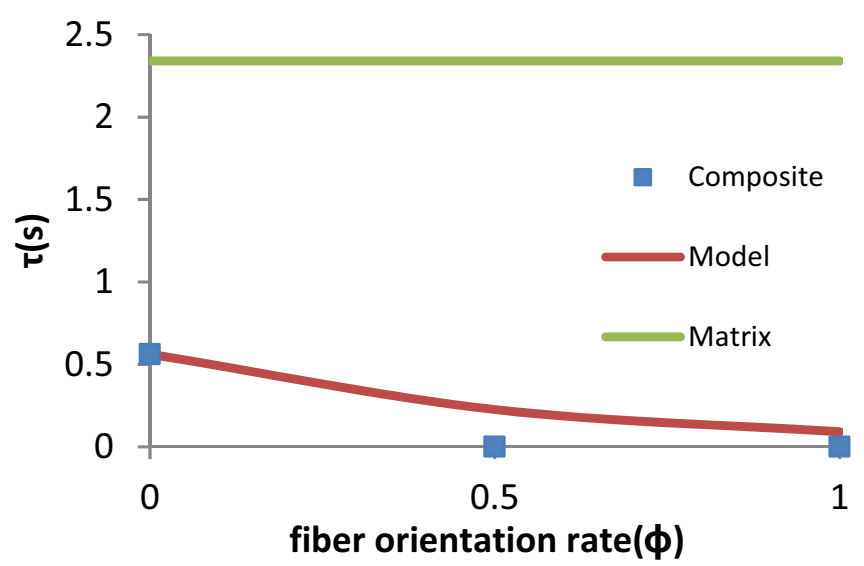

(a)

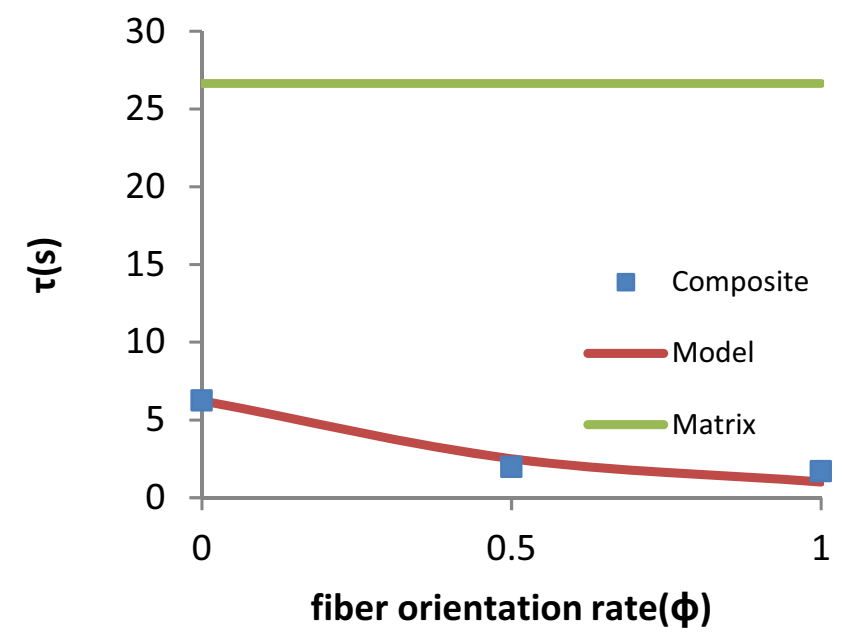

(b)

Fig. 18 Evolution of parameters as a function of the fiber ratio and the fiber orientation: $\mathbf{a}$ under and $\mathbf{b}$ above $\mathrm{Tg}$ 
- Evolution of the impact of viscosity on the material's strength as a function of the fiber ratio and the fiber orientation under the glass transition temperature:

$\delta_{e}^{F}=\delta_{e}^{m a t}(1-W t)^{\gamma} e^{-\rho F}$

where $\delta_{e}^{\text {mat }}$ is the neat PPS parameter, $W t$ is the mass fraction (here 0,4$), \gamma$ is the impact of the mass fraction on $\delta_{e^{\prime}}^{F} F$ is the fiber orientation rate with $F \varepsilon[0 ; 1]$ and $\rho$ is the weighting of the fibers orientation rate.

- Evolution of the impact of viscosity on the material strength as a function of the fiber ratio and the fiber orientation above the glass transition temperature:

$\delta^{F}=\delta^{m a t}(1-W t)^{\gamma}+p\left(1-e^{\beta F}\right)$

where $\delta^{\text {mat }}$ is the neat PPS parameter, $W t$ is the mass fraction (here 0,4$), \gamma$ is the impact of the mass fraction on $\delta^{F}, F$ is the fiber orientation rate with $F \varepsilon[0 ; 1], p$ is a corrective parameter and $\beta$ is the weighting of the fibers orientation rate.

- Evolution of the impact of viscosity on the damping of the material values as a function of the fiber ratio and the fiber orientation under and above the glass transition temperature:

$\tau_{F}=\tau_{m a t}(1-W t)^{\gamma_{\tau}} e^{-\rho F}$

where $\tau_{\text {mat }}$ is the neat PPS parameter, $W t$ is the mass fraction (here 0,4$), \gamma_{\tau}$ is the impact of the mass fraction on $\tau_{F}, F$ is the fiber orientation rate with $F \varepsilon[0 ; 1]$ and $\rho$ is the weighting of the fibers orientation rate.

It is also worth noting that in the case of the stress relaxation time, under and above $\mathrm{Tg}$, the same relation is valid with exactly the same parameters values, meaning that the glass transition increases the influence of viscosity but does not affect the shape of its curve. The analysis of the evolution of this characteristic time at the composite level shows that the parameter is far more dependent on the fiber ratio than on their orientation. This dependence can be deduced from the difference between the cross-flow direction value and the matrix value, while no particular dynamic change of its evolution was observed depending on the fiber orientation. At last, the various effects of viscosity are not influenced by the same parameters. As shown in Fig. $18, \delta$ is more sensitive to the orientation of fibers than to their mere presence, whatever the temperature. However, above $\mathrm{Tg}$, viscosity is the main factor affecting the mechanical behavior, weakening the composite's strength if the fibers are not aligned with the loading direction.

\section{Conclusion}

This paper reports the study of the influence of the hydrothermal environment on the mechanical behavior of a PPS and its composite. An experimental study was carried out, based on the equipment commonly used in the industry in order to characterize the behavior of a PPS GF40 and its matrix. It was shown that:

- The behavior of the PPS is considerably influenced by the temperature and is sensitive to absorption.

- The more the cooling liquid contains water, the more the mechanical behavior is affected, as the PPS shows more sensitivity to absorption than to the chemical aggressiveness of the cooling liquid.

- Two kinds of behavior were noted: an elasto-plastic behavior under the glass transition temperature and a visco-pseudo-plastic one above this temperature.

- Viscosity is the leading phenomenon occurring above the glass transition temperature for the PPS and can also be of great influence under $\mathrm{Tg}$, under cyclic conditions and when the stress rate is low.

- The high temperatures reduce the advantages brought by the presence of fibers.

A phenomenological model was then built to take into account these experimental observations. It was shown that the presented approach enables the description and the prediction of the mechanical response with a very good accuracy over a wide range of hydrothermal conditions. A temperature-humidity equivalence principle was highlighted for the PPS, allowing the consideration of the ageing process within the proposed model. Then, the maximal accuracy physically reachable was determined for all models using an artificial-intelligencebased model applied to the results of the experiments.

The physical reality of the parameters used in the constitutive equations was verified through experimental observations. Therefore, studying the evolution of the parameters with the adjunction and the orientation of the fibers showed that the fiber orientation has an important impact on the mechanical resistance under the glass transition temperature and a more important impact above this temperature. It was observed that the damping of the material, showing the same evolution trend under and above $\mathrm{Tg}$, was more sensitive to the fiber ratio than to their orientation.

Such results can be of great interest for the non-linear mechanical characterization of polymers, from the matrix to the composite, for a wide range of hydrothermal conditions. This analysis is required for the design of fatigue models for industrial components. 
Acknowledgements This work was supported by Pierburg Pump Technology. The authors gratefully acknowledge Solvay for providing the specimens.

\section{Compliance with ethical standards}

Conflict of interest On behalf of all the authors, the corresponding author states that there is no conflict of interest.

\section{References}

1. Piao H et al (2019) Influence of water absorption and temperature on the mechanical properties of discontinuous carbon fiber reinforced polyamide 6. Fibers Polym 20(3):611-619

2. Valentin D, Paray F, Guetta B (1987) The hygrothermal behaviour of glass fibre reinforced Pa66 composites: a study of the effect of water absorption on their mechanical properties. J Mater Sci 22(1):46-56

3. Guérin B (1994) Polyamides PA (ed) Techniques Ingénieur

4. Verdu J (2000) Action de l'eau sur les plastiques|Techniques de I'Ingénieur. AM3165

5. Christopher NSJ et al (1968) Thermal degradation of poly (phenylene sulfide) and perfluoropoly (phenylene sulfide). J Appl Polym Sci 12(4):863-870

6. Ehlers GFL, Fisch KR, Powell WR (1969) Thermal degradation of polymers with phenylene units in the chain II Sulfur-containing polyarylenes. J Polym Sci Part A-1 Polym Chem 7(10):2955-2967

7. Yu L, Bahadur S, Xue Q (1998) An investigation of the friction and wear behaviors of ceramic particle filled polyphenylene sulfide composites. Wear 214(1):54-63

8. Gyurova LA, Friedrich K (2011) Artificial neural networks for predicting sliding friction and wear properties of polyphenylene sulfide composites. Tribol Int 44(5):603-609

9. Zhao Q, Bahadur S (1998) A study of the modification of the friction and wear behavior of polyphenylene sulfide by particulate Ag2S and PbTe fillers. Wear 217(1):62-72

10. Jiang $Z$ et al (2008) Study on friction and wear behavior of polyphenylene sulfide composites reinforced by short carbon fibers and sub-micro TiO2 particles. Compos Sci Technol 68(3-4):734-742

11. Yang Y et al (2013) Morphology control of nanofillers in poly (phenylene sulfide): a novel method to realize the exfoliation of nanoclay by $\mathrm{SiO} 2$ via melt shear flow. Compos Sci Technol 75:28-34

12. Hill HW, Brady DG (1976) Properties, environmental stability, and molding characteristics of polyphenylene sulfide. Polym Eng Sci 16(12):831-835

13. López LC, Wilkes GL (1989) Non-isothermal crystallization kinetics of poly (p-phenylene sulphide). Polymer 30(5):882-887

14. Caramaro L et al (1991) Morphology and mechanical performance of polyphenylenesulfide carbon fiber composite. Polym Eng Sci 31(17):1279-1285

15. Favaloro M (2009) Properties and processes of linear polyphenylene sulfide (PPS) for continuous fiber composites aerospace applications. No. 2009-01-3242. SAE Technical Paper

16. Zhou S et al (2013) Effect of carbon fiber reinforcement on the mechanical and tribological properties of polyamide6/polyphenylene sulfide composites. Mater Des 44:493-499

17. Yýlmaz T, Sýnmazçelik T (2007) Investigation of load bearing performances of pin connected carbon/polyphenylene sulphide composites under static loading conditions. Mater Des 28(2):520-527
18. Zuo P et al (2018) Multi-scale analysis of the effect of loading conditions on monotonic and fatigue behavior of a glass fiber reinforced polyphenylene sulfide (PPS) composite. Compos B Eng 145:173-181

19. Mouhmid B, Imad A, Benseddiq N, Benmedakhene S, Maazouz A (2006) A study of the mechanical behaviour of a glass fibre reinforced polyamide 6,6: experimental investigation. Polym Test 25(4):544

20. Mulliken AD, Boyce MC (2006) Mechanics of the rate-dependent elastic-plastic deformation of glassy polymers from low to high strain rates. Int J Solids Struct 43(5):1331-1356

21. Boyce MC, Socrate S, Llana PG (2000) Constitutive model for the finite deformation stress-strain behavior of poly (ethylene terephthalate) above the glass transition. Polymer 41(6):2183-2201

22. Puffr R, Šebenda J (1967) On the structure and properties of polyamides XXVII The mechanism of water sorption in polyamides. J Polym Sci Part C Polym Symp 16(1). New York: Wiley Subscription Services, Inc., A Wiley Company

23. Launay A et al (2013) Modelling the influence of temperature and relative humidity on the time-dependent mechanical behaviour of a short glass fibre reinforced polyamide. Mech Mater 56:1-10

24. Merdas I et al (2002) Factors governing water absorption by composite matrices. Compos Sci Technol 62(4):487-492

25. Kettle GJ (1977) Variation of the glass transition temperature of nylon-6 with changing water content. Polymer 18(7):742-743

26. Praud F et al (2017) Phenomenological multi-mechanisms constitutive modelling for thermoplastic polymers, implicit implementation and experimental validation. Mech Mater 114:9-29

27. Ottosen NS, Ristinmaa M (2005) Introduction to time-dependent material behavior. In: The mechanics of constitutive modeling. Elsevier, pp 357-378

28. Ramberg W, Osgood WR (1943) Description of stress-strain curves by three parameters. Technical note 902 , National Advisory Committee for Aeronautics, Washington, DC

29. Fick A (1855) Uber diffusion. Phil Mag 10: 30-39. (reprinted in 1995 as On diffusion. J Membr Sci 100 (1855): 33-38)

30. Eftekhari M, Fatemi A (2016) Tensile behavior of thermoplastic composites including temperature, moisture, and hygrothermal effects. Polym Test 51:151-164

31. Boubakri A et al (2010) Study of UV-aging of thermoplastic polyurethane material. Mater Sci Eng A 527(7-8):1649-1654

32. Zuo $P$ et al (2019) Thermal aging effects on overall mechanical behavior of short glass fiber-reinforced polyphenylene sulfide composites. Polym Eng Sci 59(4):765-772

33. Launay A et al (2011) Cyclic behaviour of short glass fibre reinforced polyamide: experimental study and constitutive equations. Int J Plast 27(8):1267-1293

34. Chaboche JL, Lemaitre J (1990) Mechanics of solid materials. Cambridge University Press, Cambridge

35. Krairi A, Doghri I (2014) A thermodynamically-based constitutive model for thermoplastic polymers coupling viscoelasticity, viscoplasticity and ductile damage. Int J Plast 60:163-181

36. Mehdipour H, Camanho PP, Belingardi G (2019) Elasto-plastic constitutive equations for short fiber reinforced polymers. Compos B Eng 165:199-214

37. Lloyd J, Taylor JA (1994) On the temperature dependence of soil respiration. Funct Ecol 8(3):315-323

38. Noguchi K et al (2015) Homeostasis of the temperature sensitivity of respiration over a range of growth temperatures indicated by a modified Arrhenius model. New Phytol 207(1):34-42

39. Gurtin ME, Williams WO (1966) On the Clausius-Duhem inequality. No. TR-7. Brown Univ Providence RI Div of Applied Mathematics 
40. Fabre $V$ et al (2018) Time-temperature-water content equivalence on dynamic mechanical response of polyamide 6,6 . Polymer 137:22-29

41. Kitayama S, Onuki R, Yamazaki K (2014) Warpage reduction with variable pressure profile in plastic injection molding via sequential approximate optimization. Int J Adv Manuf Technol 72(5-8):827-838

42. El Kadi $\mathrm{H}$ (2006) Modeling the mechanical behavior of fiberreinforced polymeric composite materials using artificial neural networks-a review. Compos Struct 73(1):1-23
43. Mouhmid B et al (2006) A study of the mechanical behaviour of a glass fibre reinforced polyamide 6, 6: experimental investigation. Polym Test 25(4):544-552

Publisher's Note Springer Nature remains neutral with regard to jurisdictional claims in published maps and institutional affiliations. 